Pacific

Journal of

Mathematics

\title{
A COMPUTATIONAL APPROACH TO THE KOSTANT-SEKIGUCHI CORRESPONDENCE
}

Heiko Dietrich AND WiLlem A. DE GRAaF 


\title{
A COMPUTATIONAL APPROACH TO THE KOSTANT-SEKIGUCHI CORRESPONDENCE
}

\author{
Heiko Dietrich AND Willem A. DE GRAAF
}

\begin{abstract}
Let $\mathfrak{g}$ be a real form of a simple complex Lie algebra. Based on ideas of Đoković and Vinberg, we describe an algorithm to compute representatives of the nilpotent orbits of $\mathfrak{g}$ using the Kostant-Sekiguchi correspondence. Our algorithms are implemented for the computer algebra system GAP and, as an application, we have built a database of nilpotent orbits of all real forms of simple complex Lie algebras of rank at most 8 . In addition, we consider two real forms $\mathfrak{g}$ and $\mathfrak{g}^{\prime}$ of a complex simple Lie algebra $\mathfrak{g}^{c}$ with Cartan decompositions $\mathfrak{g}=\mathfrak{k} \oplus \mathfrak{p}$ and $\mathfrak{g}^{\prime}=\mathfrak{k}^{\prime} \oplus \mathfrak{p}^{\prime}$. We describe an explicit construction of an isomorphism $\mathfrak{g} \rightarrow \mathfrak{g}^{\prime}$, respecting the given Cartan decompositions, which fails if and only if $\mathfrak{g}$ and $\mathfrak{g}^{\prime}$ are not isomorphic. This isomorphism can be used to map the representatives of the nilpotent orbits of $\mathfrak{g}$ to other realizations of the same algebra.
\end{abstract}

\section{Introduction}

When considering the action of a Lie group on its Lie algebra, the question arises as to what its orbits are. This question has mainly been studied for complex simple Lie algebras $\mathfrak{g}^{c}$, with their adjoint groups $G^{c}$. Particularly the theory concerning nilpotent orbits (that is, $G^{c}$-orbits consisting of nilpotent elements) has seen many interesting developments over the past decades; we refer to [Collingwood and McGovern 1993] for a detailed account. These orbits have been classified in terms of combinatorial objects called weighted Dynkin diagrams, using a beautiful connection between nilpotent orbits and orbits of $\mathfrak{s l}_{2}$-triples. If $\mathfrak{g}^{c}$ is of classical type, then the nilpotent orbits also have been classified in terms of certain sets of partitions (of the dimension of the natural representation).

For real Lie algebras $\mathfrak{g}$, with the action of the adjoint group $G$, it is much harder to classify the nilpotent $(G$-)orbits. The main problem compared to the complex case is that a weighted Dynkin diagram can correspond to several nilpotent orbits.

The research leading to these results has received funding from the European Union's Seventh Framework Program FP7/2007-2013 under grant agreement No. 271712.

MSC2010: 17B45, 20G20.

Keywords: real Lie algebra, real nilpotent orbit, computational methods, Kostant-Sekiguchi correspondence. 
To illustrate this phenomenon consider $G^{c}=\operatorname{PSL}_{n}(\mathbb{C})$ and $G=\operatorname{PSL}_{n}(\mathbb{R})$ with their Lie algebras $\mathfrak{g}^{c}=\mathfrak{s l}_{n}(\mathbb{C})$ and $\mathfrak{g}=\mathfrak{s l}_{n}(\mathbb{R})$. The nilpotent orbits in $\mathfrak{g}^{c}$ are parametrized by partitions of $n$, whose parts correspond to the sizes of the Jordan blocks of a representative of the orbit. The nilpotent orbits in $\mathfrak{g}$ are associated with the same partitions, with the difference that the partitions with only even terms correspond to two nilpotent orbits.

More generally, the nilpotent orbits of the simple real Lie algebras of classical type have been classified in terms of combinatorial objects such as partitions or certain types of Young diagrams; see [Collingwood and McGovern 1993, Section 9.3]. For the classification in Lie algebras of exceptional types the main ingredient is the Kostant-Sekiguchi correspondence: Let $\mathfrak{g}=\mathfrak{k} \oplus \mathfrak{p}$ be a Cartan decomposition of the simple real Lie algebra $\mathfrak{g}$ with complexification $\mathfrak{g}^{c}=\mathfrak{k}^{c} \oplus \mathfrak{p}^{c}$. Let $G^{c}$ be the adjoint group of $\mathfrak{g}^{c}$ and denote by $G, K$, and $K^{c}$ the connected Lie subgroups of $G^{c}$ with corresponding Lie algebras $\mathfrak{g}, \mathfrak{k}$, and $\mathfrak{k}^{c}$, respectively. The Kostant-Sekiguchi correspondence states a one-to-one correspondence between the nilpotent orbits in $\mathfrak{g}$ and the nilpotent $K^{c}$-orbits in $\mathfrak{p}^{c}$. Although this correspondence can be described explicitly (as we will do in Section 3), it is difficult to obtain concrete representatives of nilpotent orbits in $\mathfrak{g}$. Most classification results therefore are on the complex side, that is, consider nilpotent $K^{c}$-orbits in $\mathfrak{p}^{c}$; see for example [Đoković 1988; Galina 2009; Noël 1998; 2001a; 2001b]. However, in tedious work, Đoković [1998; 1999; 2000] has used this correspondence to obtain representatives of the nilpotent orbits for each of the simple real Lie algebras of exceptional type.

The aim of this paper is to describe methods for constructing representatives of the nilpotent orbits of a real simple Lie algebra on a computer. One approach to obtain representatives is to take the existing classifications in the literature, to set up isomorphisms to the algebras given, and to map the given representatives. However, it is not straightforward to verify the correctness of the representatives given in the literature, so this approach is rather error-prone. (In fact, in each of his papers cited above, Đoković corrected some errors, due to typos, in his previous papers.) For this reason we devise algorithms that effectively carry out the Kostant-Sekiguchi correspondence. Since the correctness of each step can be checked algorithmically, we get a certified list of representatives.

1.1. Main results. We describe computational methods to achieve three aims:

(A) Construct isomorphism type representatives for all real forms of a simple complex Lie algebra.

(B) Construct representatives of all nilpotent orbits of a real form constructed in $(\mathrm{A})$.

(C) Construct an isomorphism between two given real forms of a simple complex Lie algebra. 
For computational purposes it is often needed that the Lie algebras are given by means of a multiplication table (with respect to some basis). We describe in Section 2 how to construct multiplication tables for all real forms of simple complex Lie algebras (up to isomorphism).

In Sections 3-6 we describe our algorithms to construct representatives of the nilpotent orbits of a Lie algebra constructed in (A). We combine the KostantSekiguchi correspondence (see Section 3) with the theory of carrier algebras developed in [Vinberg 1979] (see Section 4). This is inspired by Đoković's [1987] proof of the Kostant-Sekiguchi correspondence. In Section 5 we discuss the construction of so-called Chevalley systems; results obtained there will also be important for (C). In Section 6 we discuss the main computational problem for applying the KostantSekiguchi correspondence, namely, to construct a complex Cayley triple in a $K^{c}$ orbit of homogeneous $\mathfrak{s l}_{2}$-triples; we give more details in Section 3.

In order to use our lists of representatives of nilpotent orbits also in other realizations of the Lie algebras (for instance in the split real forms, in their natural representation), we devise algorithms to construct isomorphisms between real simple Lie algebras. More precisely, in Section 7 we discuss the isomorphism problem for two real forms $\mathfrak{g}$ and $\mathfrak{g}^{\prime}$ of a complex simple Lie algebra $\mathfrak{g}^{c}$. If $\mathfrak{g}=\mathfrak{k} \oplus \mathfrak{p}$ and $\mathfrak{g}^{\prime}=\mathfrak{k}^{\prime} \oplus \mathfrak{p}^{\prime}$ are given Cartan decompositions, then we describe an explicit construction of an isomorphism $\mathfrak{g} \rightarrow \mathfrak{g}^{\prime}$, respecting the given Cartan decompositions, which fails if and only if such an isomorphism does not exist.

1.2. Related work. Đoković $[1998 ; 1999 ; 2000]$ first used the Kostant-Sekiguchi correspondence to obtain representatives of nilpotent orbits for the real forms of Lie algebras of exceptional type. His methods vary somewhat from paper to paper. However, in all these publications the main idea is to start with a complex nilpotent orbit $\mathfrak{O}^{c} \subset \mathfrak{g}^{c}$ meeting $\mathfrak{g}$ nontrivially. Then some real representatives of $\mathcal{O}^{c}$ in $\mathfrak{g}$ are computed. The Kostant-Sekiguchi correspondence is used to decide whether these real representatives lie in the same $G$-orbit or not. The process stops when enough elements lying in different $G$-orbits are found. This ad hoc approach has worked for the Lie algebras of exceptional type, but there is no guarantee that it will always yield representatives of all nilpotent orbits. Furthermore, it is rather tedious to apply and difficult to translate into a systematic approach suitable for a computer.

In our approach the problem is reduced to finding a complex Cayley triple in a carrier algebra. Most carrier algebras that occur are principal and for those we have an automatic procedure for finding the triple (see Section 6.2). However, some carrier algebras are not principal, and for those we translate the problem into a set of polynomial equations that has to be solved. For dealing with the latter problem we use a simple-minded systematic technique (see Section 6.3) which turned out to work well in all our examples, which include all Lie algebras of rank at most 8. 
1.3. Computational remark. Our algorithms are implemented for the computer algebra system GAP [GAP 2012], as part of a package for doing computations with real Lie algebras, called CoReLG [CoReLG 2012]. The functions for obtaining the multiplication tables of the real simple Lie algebras in this package have been implemented by Paolo Faccin; see [Dietrich et al. 2013]. As an application, we created a database containing representatives of nilpotent orbits for all simple real forms of rank at most 8; this database will also be contained in the package CoReLG. As mentioned in the previous paragraph, we construct certain complex Cayley triples in carrier algebras. It is possible that isomorphic carrier algebras will turn up when dealing with different simple Lie algebras. To avoid dealing with the same problem twice, we have also built a database of nonprincipal carrier algebras, together with the Cayley triples that we found (see Section 6.3.1).

Our approach works uniformly for all simple real Lie algebras. However, our database is currently limited to the Lie algebras of ranks up to 8 for two reasons. Firstly, it includes all exceptional types. Secondly, in the SLA package, the current implementations of the algorithms for listing the nilpotent orbits of a $\theta$-group are not very efficient when $\theta$ is an outer automorphism. This makes it currently difficult to go beyond rank 8 when the real form is defined relative to an outer involution.

There is the question of which base field to use for the computations. The Lie algebras with which we work are defined over $\mathbb{R}$ or $\mathbb{C}$. However, we want to perform exact computations, and the field $\mathbb{Q}$ is not suitable as we often need square roots of rational numbers. For this reason we work over the field $\mathbb{Q} \sqrt{ }=\mathbb{Q}(\{\sqrt{p} \mid p$ a prime $\})$. In the Appendix we indicate how the arithmetic of that field is implemented. Since we often work in the complex Lie algebra $\mathfrak{g}^{c}$ in order to obtain results in the real Lie algebra $\mathfrak{g}$, we also use the field $\mathbb{Q} \sqrt{ }(l)$ where $\imath=\sqrt{-1} \in \mathbb{C}$.

1.4. Notation. Throughout this paper we retain the previous notation and denote by $\theta$ the Cartan involution associated with the Cartan decomposition $\mathfrak{g}=\mathfrak{k} \oplus \mathfrak{p}$. By $\mathfrak{g}^{c}=\mathfrak{k}^{c} \oplus \mathfrak{p}^{c}$ we denote the complexification of $\mathfrak{g}$, and $\sigma$ is the complex conjugation of $\mathfrak{g}^{c}$ with respect to $\mathfrak{g}$. By abuse of notation, we also denote by $\theta$ its extension to $\mathfrak{g}^{c}$. Let $G^{c}$ be the adjoint group of $\mathfrak{g}^{c}$ and denote by $G, K$, and $K^{c}$ the connected Lie subgroups of $G^{c}$ with corresponding Lie algebras $\mathfrak{g}, \mathfrak{k}$, and $\mathfrak{k}^{c}$, respectively.

\section{Constructing the Lie algebras}

The aim of this section is to describe the construction of the real forms we consider. Our computational setup is as in [de Graaf 2000]; that is, in our algorithms we suppose the Lie algebras are given by multiplication tables, usually with respect to Chevalley bases. For the sake of completeness, we first recall the relevant definitions, and then construct certain bases of all real forms (up to isomorphism) of simple complex Lie algebras. 
2.1. Canonical generators. Let $\mathfrak{g}^{c}$ be a complex semisimple Lie algebra with Cartan subalgebra $\mathfrak{h}^{c}$. Let $\Phi$ be the corresponding root system with basis of simple roots $\Delta=\left\{\alpha_{1}, \ldots, \alpha_{l}\right\}$. Then $\mathfrak{g}^{c}$ has a Chevalley basis with respect to $\Phi$; see [Humphreys 1978, Section 25.2]:

Definition 1. A basis $\left\{h_{1}, \ldots, h_{l}, x_{\alpha} \mid \alpha \in \Phi\right\}$ of $\mathfrak{g}^{c}$ is a Chevalley basis if $\left\{h_{1}, \ldots, h_{l}\right\}$ spans the Cartan subalgebra $\mathfrak{h}^{c}$ of $\mathfrak{g}^{c}$, and for all $\alpha, \beta \in \Phi$ the following hold:

- $x_{\alpha}$ spans the root space $\mathfrak{g}_{\alpha}=\left\{x \in \mathfrak{g}^{c} \mid\left[h_{i}, x\right]=\alpha\left(h_{i}\right) x\right.$ for all $\left.i\right\}$ corresponding to $\alpha$,

- $\left[x_{\alpha}, x_{-\alpha}\right]=h_{\alpha}$, where $h_{\alpha}$ is the unique element in $\left[\mathfrak{g}_{\alpha}, \mathfrak{g}_{-\alpha}\right]$ with $\alpha\left(h_{\alpha}\right)=2$; in particular, $h_{i}=h_{\alpha_{i}}$ for all $i=1, \ldots, l$,

- $\left[x_{\alpha}, x_{\beta}\right]=N_{\alpha, \beta} x_{\alpha+\beta}$ if $\alpha+\beta \in \Phi$, where $N_{\alpha, \beta} \neq 0$ is an integer with $N_{\alpha, \beta}=-N_{-\alpha,-\beta}$,

- $\left[x_{\alpha}, x_{\beta}\right]=0$ if $\alpha+\beta \notin \Phi$ and $\alpha \neq-\beta$.

Note that we see the roots in $\Phi$ as elements of the dual space $\left(\mathfrak{h}^{c}\right)^{*}$ via $\left[h, x_{\alpha}\right]=$ $\alpha(h) x_{\alpha}$. For two roots $\alpha, \beta \in \Phi$, the corresponding Cartan integer now is $\langle\alpha, \beta\rangle=$ $\alpha\left(h_{\beta}\right)$; the Cartan matrix of $\Phi$ defined by $\Delta$ is $\left(\left\langle\alpha_{i}, \alpha_{j}\right\rangle\right)_{i j}$; see [Humphreys 1978 , pp. 39, 55]. In the sequel, we usually denote by $\left\{h_{1}, \ldots, h_{l}, x_{\alpha} \mid \alpha \in \Phi\right\}$ a fixed Chevalley basis of $\mathfrak{g}^{c}$, and by $\left\{h_{i}, x_{i}, y_{i} \mid i=1, \ldots, l\right\}$ with $x_{i}=x_{\alpha_{i}}$ and $y_{i}=x_{-\alpha_{i}}$ the canonical generating set it contains:

Definition 2. A generating set $\left\{c_{i}, a_{i}, b_{i} \mid i=1, \ldots, l\right\}$ of $\mathfrak{g}^{c}$ is a canonical generating set if for all $i, j \in\{1, \ldots, l\}$ the following hold:

- $c_{i} \in \mathfrak{h}^{c}, a_{i} \in \mathfrak{g}_{\alpha_{i}}$, and $b_{i} \in \mathfrak{g}_{-\alpha_{i}}$,

- $\left[c_{i}, c_{j}\right]=0$ and $\left[a_{i}, b_{j}\right]=\delta_{i j} c_{i}$, where $\delta_{i j}$ is the Kronecker delta,

- $\left[c_{i}, a_{j}\right]=\left\langle\alpha_{j}, \alpha_{i}\right\rangle a_{j}$ and $\left[c_{i}, b_{j}\right]=-\left\langle\alpha_{j}, \alpha_{i}\right\rangle b_{j}$.

Let $\left\{c_{i}^{\prime}, a_{i}^{\prime}, b_{i}^{\prime} \mid i=1, \ldots, l\right\}$ be a second canonical generating set of $\mathfrak{g}^{c}$, possibly relative to a different basis of simple roots $\Delta^{\prime}$. If $\Delta$ and $\Delta^{\prime}$ define the same Cartan matrix, then there exists a unique automorphism of $\mathfrak{g}^{c}$ which maps $\left(c_{i}, a_{i}, b_{i}\right)$ to $\left(c_{i}^{\prime}, a_{i}^{\prime}, b_{i}^{\prime}\right)$ for every $i=1, \ldots, l$; see [Jacobson 1962, Chapter IV, Theorem 3]. We freely use this property throughout the paper. Also, if $\Phi$ and $l$ follow from the context, then we write $\left\{h_{i}, x_{\alpha} \mid \alpha, i\right\}$ and $\left\{h_{i}, x_{i}, y_{i} \mid i\right\}$ for the Chevalley basis and canonical generating set. We end this section with a proposition, which yields a straightforward algorithm to obtain a canonical generating set. For its proof, as well as the algorithm, we refer to [de Graaf 2000, Section 5.11].

Proposition 3. For $i=1, \ldots$, l let $a_{i} \in \mathfrak{g}_{\alpha_{i}}$ and $b_{i} \in \mathfrak{g}_{-\alpha_{i}}$, and write $c_{i}=\left[a_{i}, b_{i}\right]$. If $\left[c_{i}, a_{i}\right]=2 a_{i}$ for all $i$, then $\left\{c_{i}, a_{i}, b_{i} \mid i\right\}$ is a canonical generating set of $\mathfrak{g}^{c}$. 
2.2. Real forms. We now turn to the construction of the real forms of a complex semisimple Lie algebra $\mathfrak{g}^{c}$; without loss of generality, we may assume that $\mathfrak{g}^{c}$ is simple. We continue to use the notation of Section 2.1; that is, $\mathfrak{h}^{c}$ is a Cartan subalgebra of $\mathfrak{g}^{c}$ with root system $\Phi$, having basis of simple roots $\Delta=\left\{\alpha_{1}, \ldots, \alpha_{l}\right\}$. Let $\left\{h_{i}, x_{\alpha} \mid i, \alpha\right\}$ and $\left\{h_{i}, x_{i}, y_{i} \mid i\right\}$ be a corresponding Chevalley basis and canonical generating set. Recall that a real Lie algebra $\mathfrak{g}^{\prime}$ is a real form of $\mathfrak{g}^{c}$ if $\mathfrak{g}^{c}=\mathfrak{g}^{\prime} \oplus \mathfrak{l} \mathfrak{g}^{\prime}$ as real vectorspaces.

2.2.1. Real forms defined by involutions. It is proved in [Onishchik 2004, Theorem 3.1] that the real subalgebra $\mathfrak{u}$ of $\mathfrak{g}^{c}$ defined as

$$
\mathfrak{u}=\operatorname{Span}_{\mathbb{R}}\left(\left\{l h_{1}, \ldots, l h_{l}, x_{\alpha}-x_{-\alpha}, l\left(x_{\alpha}+x_{-\alpha}\right) \mid \alpha \in \Phi^{+}\right\}\right)
$$

is a (compact) real form of $\mathfrak{g}^{c}$. Let $\tau$ be the corresponding real structure; that is, $\tau: \mathfrak{g}^{c} \rightarrow \mathfrak{g}^{c}$ is the complex conjugation of $\mathfrak{g}^{c}=\mathfrak{u} \oplus \imath \mathfrak{u}$ with respect to $\mathfrak{u}$. This implies that $\tau\left(x_{\alpha}\right)=-x_{-\alpha}$ for all $\alpha \in \Phi$; in particular, for all $i=1, \ldots, l$ we have

$$
\tau\left(h_{i}\right)=-h_{i}, \quad \tau\left(x_{i}\right)=-y_{i}, \quad \text { and } \quad \tau\left(y_{i}\right)=-x_{i} .
$$

It follows from [Onishchik 2004, Theorem 3.2] that, up to isomorphism, every real form of $\mathfrak{g}^{c}$ is constructed as follows: Let $\theta$ be an involutive automorphism of $\mathfrak{g}^{c}$ commuting with $\tau$. Then $\mathfrak{u}=\mathfrak{u}_{0} \oplus \mathfrak{u}_{1}$, where $\mathfrak{u}_{i}$ is the eigenspace of $\theta$ in $\mathfrak{u}$ with eigenvalue $(-1)^{i}$, and the real form defined by $\mathfrak{u}$ and $\theta$ is

$$
\mathfrak{g}=\mathfrak{g}(\theta, \mathfrak{u})=\mathfrak{k} \oplus \mathfrak{p} \quad \text { with } \mathfrak{k}=\mathfrak{u}_{0} \text { and } \mathfrak{p}=\imath \mathfrak{u}_{1} .
$$

This decomposition of $\mathfrak{g}$ is a Cartan decomposition whose Cartan involution is the restriction of $\theta$ to $\mathfrak{g}$; see [Onishchik 2004, Section 5]. We denote by $\sigma: \mathfrak{g}^{c} \rightarrow \mathfrak{g}^{c}$ the complex conjugation of $\mathfrak{g}^{c}=\mathfrak{g} \oplus \mathfrak{l g}$ relative to $\mathfrak{g}$.

Two such real forms $\mathfrak{g}(\theta, \mathfrak{u})$ and $\mathfrak{g}\left(\theta^{\prime}, \mathfrak{u}\right)$ are isomorphic if and only if $\theta$ and $\theta^{\prime}$ are conjugate in $\operatorname{Aut}\left(\mathfrak{g}^{c}\right)$. The finite order automorphisms of $\mathfrak{g}^{c}$ are, up to conjugacy, classified by so-called Kac diagrams; see [Vinberg et al. 1990, Section 3.3.7] or [Helgason 1978, Section X.5]. By running through these diagrams we can efficiently construct all involutions of $\mathfrak{g}^{c}$ up to conjugacy, and hence all real forms of $\mathfrak{g}^{c}$ up to isomorphism.

2.2.2. Real forms of inner type. Let $\theta$ be an inner involutive automorphism of $\mathfrak{g}^{c}$. Up to conjugacy, $\theta$ maps $\left(h_{i}, x_{i}, y_{i}\right)$ to $\left(h_{i}, \lambda_{i} x_{i}, \lambda_{i}^{-1} y_{i}\right)$ with $\lambda_{i} \in\{ \pm 1\}$ for all $i$. Clearly, such an automorphism commutes with $\tau$, and bases of $\mathfrak{k}$ and $\mathfrak{p}$ in $\mathfrak{g}=$ $\mathfrak{g}(\theta, \mathfrak{u})=\mathfrak{k} \oplus \mathfrak{p}$ are

$$
\begin{aligned}
& \mathscr{K}=\left\{x_{\alpha}-x_{-\alpha}, l\left(x_{\alpha}+x_{-\alpha}\right) \mid \alpha \in \Phi^{+} \text {with } \theta\left(x_{\alpha}\right)=x_{\alpha}\right\} \cup\left\{\imath h_{1}, \ldots, l h_{l}\right\}, \\
& \mathscr{P}=\left\{l\left(x_{\alpha}-x_{-\alpha}\right), x_{\alpha}+x_{-\alpha} \mid \alpha \in \Phi^{+} \text {with } \theta\left(x_{\alpha}\right)=-x_{\alpha}\right\} .
\end{aligned}
$$


We define $\mathfrak{g}$ by the multiplication table constructed via the basis $\mathscr{B}=\mathscr{K} \cup \mathscr{P}$. We note that $\left\{l h_{1}, \ldots, l h_{l}\right\}$ spans a Cartan subalgebra $\mathfrak{h}_{0}$ of $\mathfrak{k}$, which is also a Cartan subalgebra of $\mathfrak{g}$. It is straightforward to see that $\sigma\left(x_{\alpha}\right)=-x_{-\alpha}$ if $\theta\left(x_{\alpha}\right)=x_{\alpha}$, and $\sigma\left(x_{\alpha}\right)=x_{-\alpha}$ if $\theta\left(x_{\alpha}\right)=-x_{\alpha}$.

2.2.3. Real forms of outer type. Let $\theta$ be an outer involutive automorphism of $\mathfrak{g}^{c}$. Up to conjugacy, $\theta=\varphi \circ \chi$, where $\varphi$ is an involutive diagram automorphism and $\chi$ is an inner involutive automorphism; clearly, $\chi$ and $\varphi$ commute. As above, we can assume that $\chi$ maps $\left(h_{i}, x_{i}, y_{i}\right)$ to $\left(h_{i}, \lambda_{i} x_{i}, \lambda_{i}^{-1} y_{i}\right)$ with $\lambda_{i} \in\{ \pm 1\}$ for all $i$. Further, $\varphi$ maps $\left(h_{i}, x_{i}, y_{i}\right)$ to $\left(h_{\pi(i)}, x_{\pi(i)}, y_{\pi(i)}\right)$ for all $i$, where $\pi$ is an involutive permutation of $\{1, \ldots, l\}$ with $\left(\left\langle\alpha_{j}, \alpha_{i}\right\rangle\right)_{i j}=\left(\left\langle\alpha_{\pi(j)}, \alpha_{\pi(i)}\right\rangle\right)_{i j}$; note that $\left\{h_{\pi(i)}, x_{\pi(i)}, y_{\pi(i)} \mid i\right\}$ also is a canonical generating set, and $\lambda_{\pi(i)}=\lambda_{i}$ since $\chi$ and $\varphi$ commute. The permutation $\pi$ induces an automorphism of $\Phi$, which we also denote by $\varphi$; that is, $\varphi\left(\alpha_{i}\right)=\alpha_{\pi(i)}$.

Let $\mathfrak{g}=\mathfrak{g}(\theta, \mathfrak{u})=\mathfrak{k} \oplus \mathfrak{p}$. We now determine bases $\mathscr{K}$ and $\mathscr{P}$ for $\mathfrak{k}$ and $\mathfrak{p}$, and, as before, define $\mathfrak{g}$ by the multiplication table constructed via $\mathscr{B}=\mathscr{K} \cup \mathscr{P}$. Since $\mathfrak{g}^{c}$ admits outer automorphisms, it is of type $A, D$, or $E_{6}$, in particular, simply laced; see [Onishchik 2004, Table 1]. We first consider the case where $\mathfrak{g}^{c}$ is not of type $A_{l}$ with $l$ even. In this case there exists a Chevalley basis $\left\{h_{i}, \hat{x}_{\alpha} \mid i, \alpha\right\}$ such that, when defining $\hat{N}_{\alpha, \beta}$ by $\left[\hat{x}_{\alpha}, \hat{x}_{\beta}\right]=\hat{N}_{\alpha, \beta} \hat{x}_{\alpha+\beta}$, we have $\hat{N}_{\varphi(\alpha), \varphi(\beta)}=\hat{N}_{\alpha, \beta}$ for all $\alpha, \beta \in \Phi$; see [Kac 1990, Section 7.9] or [de Graaf 2000, Section 5.15]. (This result does not hold if $\mathfrak{g}^{c}$ is of type $A_{l}$ with $l$ even; we consider this case in the following section.) Induction on the height of $\alpha$ now proves that $\varphi\left(\hat{x}_{\alpha}\right)=\hat{x}_{\varphi(\alpha)}$ for all $\alpha \in \Phi$. Thus, if $\varphi(\alpha)=\alpha$, then $\varphi$ acts as the identity on $\mathfrak{g}_{\alpha}$, which implies that $\varphi\left(x_{\alpha}\right)=x_{\alpha}$.

For $\alpha \in \Phi$ define

$$
v_{\alpha}=x_{\alpha}-\varphi\left(x_{\alpha}\right) \quad \text { and } \quad u_{\alpha}=\left\{\begin{array}{cc}
x_{\alpha} & \text { if } \varphi(\alpha)=\alpha \\
x_{\alpha}+\varphi\left(x_{\alpha}\right) & \text { if } \varphi(\alpha) \neq \alpha
\end{array}\right.
$$

Let $\Psi^{+}$be the set consisting of all $\alpha \in \Phi^{+}$such that $\varphi(\alpha)=\alpha$, along with one element of each pair $(\alpha, \varphi(\alpha))$ where $\varphi(\alpha) \neq \alpha$. Let $\Phi \subseteq\{1, \ldots, l\}$ be a set of representatives of the $\pi$-orbits on $\{1, \ldots, l\}$ of length 2 . Now we define $\mathscr{K}$ as the union of the three sets

$$
\begin{gathered}
\mathscr{H}_{0}=\left\{l h_{i} \mid i=1, \ldots, l \text { with } \pi(i)=i\right\} \cup\left\{l\left(h_{i}+h_{\pi(i)}\right) \mid i \in \mathscr{I}\right\}, \\
\left\{u_{\alpha}-u_{-\alpha}, l\left(u_{\alpha}+u_{-\alpha}\right) \mid \alpha \in \Psi^{+} \text {with } \chi\left(x_{\alpha}\right)=x_{\alpha}\right\}, \quad \text { and } \\
\left\{v_{\alpha}-v_{-\alpha}, l\left(v_{\alpha}+v_{-\alpha}\right) \mid \alpha \in \Psi^{+} \text {with } \chi\left(x_{\alpha}\right)=-x_{\alpha} \text { and } \varphi(\alpha) \neq \alpha\right\} ;
\end{gathered}
$$

note that, if $\varphi(\alpha)=\alpha$ and $\chi\left(x_{\alpha}\right)=x_{\alpha}$, then $\theta\left(x_{\alpha}\right)=x_{\alpha}$, whence $u_{\alpha}-u_{-\alpha}$ and $\imath\left(u_{\alpha}+u_{-\alpha}\right)$ lie in $\mathfrak{k}$. We define $\mathscr{P}$ to be the union of 
$\left\{h_{i}-h_{\pi(i)} \mid i \in \mathscr{I}\right\}, \quad\left\{l\left(u_{\alpha}-u_{-\alpha}\right), u_{\alpha}+u_{-\alpha} \mid \alpha \in \Psi^{+}\right.$with $\left.\chi\left(x_{\alpha}\right)=-x_{\alpha}\right\}$, and $\left\{l\left(v_{\alpha}-v_{-\alpha}\right), v_{\alpha}+v_{-\alpha} \mid \alpha \in \Psi^{+}\right.$with $\chi\left(x_{\alpha}\right)=x_{\alpha}$ and $\left.\varphi(\alpha) \neq \alpha\right\}$.

It is straightforward to verify that $\mathscr{K}$ and $\mathscr{P}$ are bases of $\mathfrak{k}$ and $\mathfrak{p}$. Further, $\mathscr{H}_{0}$ spans a Cartan subalgebra $\mathfrak{h}_{0}$ of $\mathfrak{k}$, but this time the complexification $\mathfrak{h}_{0}^{c}$ is not a Cartan subalgebra of $\mathfrak{g}^{c}$. We have $\sigma\left(u_{\alpha}\right)=-u_{-\alpha}$ and $\sigma\left(v_{\alpha}\right)=v_{-\alpha}$ if $\chi\left(x_{\alpha}\right)=x_{\alpha}$, and $\sigma\left(u_{\alpha}\right)=u_{-\alpha}$ and $\sigma\left(v_{\alpha}\right)=-v_{-\alpha}$ otherwise.

Remark 4. We consider the weight space decomposition of $\mathfrak{g}^{c}$ with respect to $\mathfrak{h}_{0}^{c}$ and show that each weight space in $\mathfrak{k}^{c}$ and $\mathfrak{p}^{c}$ (corresponding to a nonzero weight) is 1-dimensional. Note that $\varphi$ fixes $\mathfrak{h}_{0}^{c}$ pointwise and, if $h \in \mathfrak{h}_{0}^{c}$, then $\alpha_{i}(h) \varphi\left(x_{i}\right)=$ $\varphi\left(\left[h, x_{i}\right]\right)=\left[h, \varphi\left(x_{i}\right)\right]=\varphi\left(\alpha_{i}\right)(h) \varphi\left(x_{i}\right)$ for all $i$, implying that $\alpha(h)=\varphi(\alpha)(h)$ for all $\alpha \in \Phi$. Now write $\Psi=\Psi^{+} \cup\left(-\Psi^{+}\right)$and define $\Psi_{0}=\left\{\left.\alpha\right|_{\mathfrak{h}_{0}^{c}} \mid \alpha \in \Psi\right\}$ as a subset of $\left(\mathfrak{h}_{0}^{c}\right)^{\star}$. Consider the simple Lie algebra $\mathfrak{l}=\left\{x \in \mathfrak{g}^{c} \mid \varphi(x)=x\right\}$; see [Kac 1990, Section 7.9]. It is easy to verify that for all $\alpha \in \Psi$ we have $u_{\alpha} \in \mathfrak{l}$, and, further, if $h \in \mathfrak{h}_{0}^{c}$, then $\left[h, u_{\alpha}\right]=\alpha(h) u_{\alpha}$. Since $\mathfrak{l}$ is simple, this proves that the root space decomposition of $\mathfrak{l}$ with respect to $\mathfrak{h}_{0}^{c}$ is $\mathfrak{l}=\mathfrak{h}_{0}^{c} \oplus \bigoplus_{\alpha \in \Psi} \mathfrak{l}_{\alpha}$, where $\mathfrak{l}_{\alpha}$ is spanned by $u_{\alpha}$; in particular, $|\Psi|=\left|\Psi_{0}\right|$. So we have the $\mathfrak{h}_{0}^{c}$-weight space decompositions

$\mathfrak{k}^{c}=\mathfrak{h}_{0}^{c} \oplus \bigoplus_{\alpha \in \Psi_{0}} \mathfrak{k}_{\alpha}^{c}, \quad \mathfrak{p}^{c}=\operatorname{Span}_{\mathbb{C}}\left(\left\{h_{i}-h_{\pi(i)} \mid i=1, \ldots, l\right.\right.$ with $\left.\left.\pi(i) \neq i\right\}\right) \oplus \bigoplus_{\alpha \in \Psi_{0}} \mathfrak{p}_{\alpha}^{c}$,

where each $\mathfrak{k}_{\alpha}^{c}=\left\{x \in \mathfrak{k}^{c} \mid[h, x]=\alpha(h) x\right.$ for all $\left.h \in \mathfrak{h}_{0}^{c}\right\}$ (and similarly $\mathfrak{p}_{\alpha}^{c}$ ) has dimension at most one. More precisely, if $\alpha \in \Phi$ and $\bar{\alpha}=\left.\alpha\right|_{\mathfrak{h}_{0}^{c}}$, then the following hold:

- if $\varphi(\alpha) \neq \alpha$ and $\chi\left(x_{\alpha}\right)=x_{\alpha}$, then $\mathfrak{k}_{\bar{\alpha}}^{c}=\operatorname{Span}_{\mathbb{C}}\left(u_{\alpha}\right)$ and $\mathfrak{p}_{\bar{\alpha}}^{c}=\operatorname{Span}_{\mathbb{C}}\left(v_{\alpha}\right)$,

- if $\varphi(\alpha) \neq \alpha$ and $\chi\left(x_{\alpha}\right) \neq x_{\alpha}$, then $\mathfrak{k}_{\bar{\alpha}}^{c}=\operatorname{Span}_{\mathbb{C}}\left(v_{\alpha}\right)$ and $\mathfrak{p}_{\bar{\alpha}}^{c}=\operatorname{Span}_{\mathbb{C}}\left(u_{\alpha}\right)$,

- if $\varphi(\alpha)=\alpha$ and $\chi\left(x_{\alpha}\right)=x_{\alpha}$, then $\mathfrak{k}_{\bar{\alpha}}^{c}=\operatorname{Span}_{\mathbb{C}}\left(u_{\alpha}\right)$ and $\mathfrak{p}_{\bar{\alpha}}^{c}=0$,

- if $\varphi(\alpha)=\alpha$ and $\chi\left(x_{\alpha}\right) \neq x_{\alpha}$, then $\mathfrak{k}_{\bar{\alpha}}^{c}=0$ and $\mathfrak{p}_{\bar{\alpha}}^{c}=\operatorname{Span}_{\mathbb{C}}\left(u_{\alpha}\right)$.

2.2.4. Real forms of $A_{l}, l$ even, of outer type. It remains to consider the case where $\mathfrak{g}^{c}$ is of type $A_{l}$ with $l=2 m$ even; we use the notation of the previous section. Up to conjugacy, we can assume that $\chi$ is the identity; thus $\theta=\varphi$ is the unique diagram automorphism. (This follows directly from looking at the possible Kac diagrams of an outer involution in this case.) Since $\mathfrak{g}^{c}$ is simply laced, $N_{\alpha, \beta}= \pm 1$ for all $\alpha, \beta \in \Phi$ with $\alpha+\beta \in \Phi$, and induction on the height of $\alpha$ proves that $\varphi\left(x_{\alpha}\right)= \pm x_{\varphi(\alpha)}$ for all $\alpha \in \Phi$. By [Kac 1990, Section 7.10], there is a Chevalley basis of $\mathfrak{g}^{c}$ such that $\varphi\left(x_{\alpha}\right)=-x_{\alpha}$ for all $\alpha \in \Phi$ with $\varphi(\alpha)=\alpha$. Let $\mathfrak{g}=\mathfrak{g}(\theta, \mathfrak{u})=\mathfrak{k} \oplus \mathfrak{p}$. A basis of $\mathfrak{k}$ is the set $\mathscr{K}$ defined as the union of

$\mathscr{H}_{0}=\left\{l\left(h_{i}+h_{\pi(i)}\right) \mid i \in \mathscr{I}\right\} \quad$ and $\left\{u_{\alpha}-u_{-\alpha}, l\left(u_{\alpha}+u_{-\alpha}\right) \mid \alpha \in \Psi^{+}\right.$with $\left.\varphi(\alpha) \neq \alpha\right\} ;$ 
note that $|\mathscr{F}|=m$ since $\pi$ acts fixed-point freely on $\{1, \ldots, 2 m\}$. A basis $\mathscr{P}$ of $\mathfrak{p}$ is the union of

$$
\begin{gathered}
\left\{h_{i}-h_{\pi(i)} \mid i \in \Phi\right\}, \quad\left\{l\left(u_{\alpha}-u_{-\alpha}\right), u_{\alpha}+u_{-\alpha} \mid \alpha \in \Psi^{+} \text {with } \varphi(\alpha)=\alpha\right\}, \\
\text { and } \quad\left\{l\left(v_{\alpha}-v_{-\alpha}\right), v_{\alpha}+v_{-\alpha} \mid \alpha \in \Psi^{+} \text {with } \varphi(\alpha) \neq \alpha\right\} .
\end{gathered}
$$

Again, $\mathscr{H}_{0}$ spans a Cartan subalgebra $\mathfrak{h}_{0}$ of $\mathfrak{k}$, and $\mathfrak{h}_{0}^{c}$ is not a Cartan subalgebra of $\mathfrak{g}^{c}$. We obtain weight space decompositions of $\mathfrak{k}^{c}$ and $\mathfrak{p}^{c}$ as in Section 2.2.3. All nonzero weight spaces with respect to $\mathfrak{h}_{0}^{c}$ are 1 -dimensional and spanned by a $u_{\alpha}$ or $v_{\alpha}$. Again, $\sigma\left(u_{\alpha}\right)=u_{-\alpha}$ and $\sigma\left(v_{\alpha}\right)=v_{-\alpha}$.

\section{Kostant-Sekiguchi correspondence}

Let $\mathfrak{g}^{c}$ be a complex semisimple Lie algebra with real form $\mathfrak{g}=\mathfrak{k} \oplus \mathfrak{p}$, associated complex conjugation $\sigma$, and Cartan involution $\theta$. Recall that we denote by $G$ and $K^{c}$ the connected Lie subgroups of the adjoint group $G^{c}$ of $\mathfrak{g}^{c}$ with Lie algebras $\mathfrak{g}$ and $\mathfrak{k}^{c}$, respectively. The Kostant-Sekiguchi correspondence is a one-toone correspondence between the nilpotent $G$-orbits in $\mathfrak{g}$ and the nilpotent $K^{c}$-orbits in $\mathfrak{p}^{c}$. The latter orbits can be constructed using the algorithms in [de Graaf 2011; 2012]; note that $K^{c}$, together with its action on $\mathfrak{p}^{c}$, is a so-called $\theta$-group. An implementation of the Kostant-Sekiguchi correspondence would therefore allow us to construct the nilpotent $G$-orbits in $\mathfrak{g}$.

We now describe this correspondence in more detail. Its proof has been completed independently in [Đoković 1987] and [Sekiguchi 1987]; here we follow the description in the first of these papers, and refer the reader to it for an historical account and (references to) proofs. First, we need some notation. The following definitions are as in [Đoković 1987] with the exception that our " $f$ " has been replaced by " $-f$ ". An $\mathfrak{s l}_{2}$-triple in $\mathfrak{g}$ (or $\left.\mathfrak{g}^{c}\right)$ is a triple $(f, h, e)$ of elements in $\mathfrak{g}\left(\right.$ or $\left.\mathfrak{g}^{c}\right)$ such that $[h, e]=2 e,[h, f]=-2 f$, and $[e, f]=h$. The characteristic (element) of this triple is $h$.

Definition 5. (a) An $\mathfrak{s l}_{2}$-triple $(f, h, e)$ in $\mathfrak{g}^{c}$ is homogeneous if $e, f \in \mathfrak{p}^{c}$ and $h \in \mathfrak{k}^{c}$.

(b) An $\mathfrak{s l}_{2}$-triple $(f, h, e)$ in $\mathfrak{g}^{c}$ is a complex Cayley triple if it is homogeneous and $\sigma(e)=f$.

(c) An $\mathfrak{s l}_{2}$-triple $(f, h, e)$ in $\mathfrak{g}$ is a real Cayley triple if $\theta(e)=-f$.

The Kostant-Sekiguchi correspondence can now be stated as in Figure 1, where all maps are bijections. We provide some details. Every nonzero nilpotent $e \in \mathfrak{p}^{c}$ lies in some homogeneous $\mathfrak{s l}_{2}$-triple $(f, h, e)$ of $\mathfrak{g}^{c}$, and the projection $(f, h, e) \mapsto e$ induces a bijection between the $K^{c}$-orbits of homogeneous $\mathfrak{s l}_{2}$-triples in $\mathfrak{g}^{c}$ and the $K^{c}$-orbits of nonzero nilpotent elements in $\mathfrak{p}^{c}$; let $\varphi_{1}$ denote the inverse of this 
bijection. Every $K^{c}$-orbit of homogeneous $\mathfrak{s l}_{2}$-triples in $\mathfrak{g}^{c}$ contains a complex Cayley triple and, conversely, every $K$-orbit of complex Cayley triples in $\mathfrak{g}^{c}$ is contained in a unique $K^{c}$-orbit of homogeneous $\mathfrak{s l}_{2}$-triples in $\mathfrak{g}^{c}$. Thus, inclusion gives rise to a bijection between the $K$-orbits of complex Cayley triples and the $K^{c}$-orbits of homogeneous $\mathfrak{s l}_{2}$-triples in $\mathfrak{g}^{c}$; again, let $\varphi_{2}$ denote the inverse of this bijection. Let $(f, h, e)$ be a real Cayley triple. Then its Cayley transform is the triple

$$
\left(\frac{1}{2}(l e+l f+h), l(e-f), \frac{1}{2}(-l e-l f+h)\right),
$$

which is a complex Cayley triple. The inverse Cayley transform maps a complex Cayley triple $(f, h, e)$ to the real Cayley triple

$$
\left(\frac{1}{2} l(e-f+h), e+f, \frac{1}{2} l(e-f-h)\right) .
$$

Taking inverse Cayley transforms induces a bijection $\varphi_{3}$ between the $K$-orbits of complex Cayley triples in $\mathfrak{g}^{c}$ and the $K$-orbits of real Cayley triples in $\mathfrak{g}$. The projection $(f, h, e) \mapsto e$ yields a bijection $\varphi_{4}$ between these $K$-orbits of real Cayley triples and the $G$-orbits of nonzero nilpotent elements in $\mathfrak{g}$. In conclusion, the Kostant-Sekiguchi correspondence states that $\varphi_{4} \circ \varphi_{3} \circ \varphi_{2} \circ \varphi_{1}$ is a bijection.

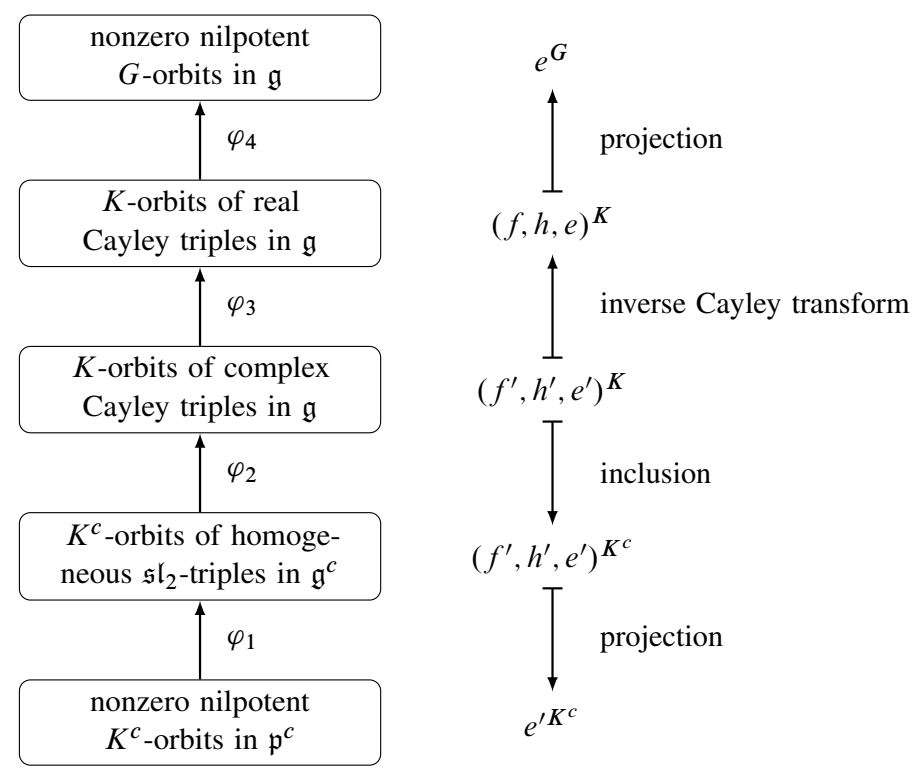

Figure 1. Kostant-Sekiguchi correspondence.

Using the algorithms of [de Graaf 2011;2012], we can compute all $K^{c}$-orbits of homogeneous $\mathfrak{s l}_{2}$-triples in $\mathfrak{g}^{c}$, which also gives us the bijection $\varphi_{1}$. A realization of 
the map $\varphi_{4} \circ \varphi_{3}$ is straightforward. Thus, computationally, it remains to realize $\varphi_{2}$, that is:

Main Problem. Find a complex Cayley triple in a $K^{c}$-orbit of homogeneous $\mathfrak{s l}_{2}-$ triples.

We discuss our approach to this problem in Section 6. For this purpose, we require some preliminary results; the subsequent sections therefore introduce carrier algebras and Chevalley systems.

\section{Carrier algebras}

We briefly review the theory of carrier algebras as developed in [Vinberg 1979]. In general, carrier algebras are connected to $\mathbb{Z}_{m}$-graded Lie algebras. Since we exclusively deal with $\mathbb{Z}_{2}$-gradings (coming from Cartan decompositions), we only consider this case here.

Let $\mathfrak{g}=\mathfrak{k} \oplus \mathfrak{p}$ be as in Section 2.2, and consider the $\mathbb{Z}_{2}$-grading $\mathfrak{g}^{c}=\mathfrak{g}_{0}^{c} \oplus \mathfrak{g}_{1}^{c}$, where $\mathfrak{g}_{0}^{c}=\mathfrak{k}^{c}$ and $\mathfrak{g}_{1}^{c}=\mathfrak{p}^{c}$. Recall that $G_{0}=K^{c}$ is the connected Lie subgroup of $G^{c}$ with Lie algebra $\mathfrak{g}_{0}^{c}$. Let $e \in \mathfrak{g}_{1}^{c}$ be nilpotent, and consider the normalizer $N_{0}(e)=\left\{x \in \mathfrak{g}_{0}^{c} \mid[x, e]=\lambda e\right.$ for some $\left.\lambda \in \mathbb{C}\right\}$. Let $\mathfrak{t}$ be a maximal torus of $N_{0}(e)$, that is, a maximal abelian subalgebra consisting of semisimple elements, and let $\mu \in \mathfrak{t}^{*}$ be defined by $[t, e]=\mu(t) e$ for $t \in \mathfrak{t}$. Let $\mathfrak{a}^{c}=\bigoplus_{k \in \mathbb{Z}} \mathfrak{a}_{k}$ be the $\mathbb{Z}$-graded Lie algebra defined by

$$
\mathfrak{a}_{k}=\left\{x \in \mathfrak{g}_{k \bmod 2}^{c} \mid[t, x]=k \mu(t) x \text { for all } t \in \mathfrak{t}\right\} .
$$

The carrier algebra of $e$ is the commutator algebra of $\mathfrak{a}^{c}$ with the inherited $\mathbb{Z}$ grading; that is,

$$
\mathfrak{s}^{c}=\mathfrak{s}(e, \mathfrak{t})=\bigoplus_{k \in \mathbb{Z}} \mathfrak{s}_{k}=\left[\mathfrak{a}^{c}, \mathfrak{a}^{c}\right]
$$

As shown in [Vinberg 1979], it has the following properties:

- $\mathfrak{s}^{c}$ is semisimple with $\operatorname{dim} \mathfrak{s}_{0}=\operatorname{dim} \mathfrak{s}_{1}$,

- $\mathfrak{s}^{c}$ is not a proper subalgebra of a $\mathbb{Z}$-graded semisimple subalgebra of $\mathfrak{g}^{c}$ of the same rank,

- $\mathfrak{s}_{k} \subseteq \mathfrak{k}^{c}$ if $k$ is even, and $\mathfrak{s}_{k} \subseteq \mathfrak{p}^{c}$ otherwise,

- $\mathfrak{s}^{c}$ is normalized by a Cartan subalgebra of $\mathfrak{g}_{0}^{c}$.

Moreover, $e \in \mathfrak{s}_{1}$ is in general position; that is, $\left[\mathfrak{s}_{0}, e\right]=\mathfrak{s}_{1}$; every element in $\mathfrak{s}_{1}$ in general position is $G_{0}$-conjugate to $e$. If $(f, h, e)$ is a homogeneous $\mathfrak{s l}_{2}$-triple in $\mathfrak{s}^{c}$, that is, $h \in \mathfrak{s}_{0}$ and $f \in \mathfrak{s}_{-1}$, then $h / 2$ is the unique defining element of $\mathfrak{s}^{c}$; that is, for all $k$,

$$
\mathfrak{s}_{k}=\left\{x \in \mathfrak{s}^{c} \mid\left[\frac{h}{2}, x\right]=k x\right\} .
$$


Since all maximal tori of $N_{0}(e)$ are conjugate, this yields a bijection between the nilpotent $G_{0}$-orbits in $\mathfrak{g}_{1}^{c}$ and the $G_{0}$-conjugacy classes of $\mathbb{Z}$-graded subalgebras with the above properties. This bijection can be used for an algorithm to list the nilpotent $G_{0}$-orbits in $\mathfrak{g}_{1}^{c}$; see [de Graaf 2011; Littelmann 1996].

Remark 6. Suppose $(f, h, e)$ is a homogeneous $\mathfrak{s l}_{2}$-triple in $\mathfrak{g}^{c}$ and let $\mathfrak{s}^{c}=\mathfrak{s}^{c}(e, \mathfrak{t})$ be a carrier algebra. Since $h \in N_{0}(e)$, we can choose a torus containing $h$; thus $h \in \mathfrak{s}^{c}$. By the Jacobson-Morozov theorem (see [Knapp 2002, Theorem X.10.3]) there is $f^{\prime} \in \mathfrak{s}^{c}$ such that $\left(f^{\prime}, h, e\right)$ is an $\mathfrak{s l}_{2}$-triple in $\mathfrak{s}^{c}$, hence also in $\mathfrak{g}^{c}$. The same theorem shows $f=f^{\prime}$; thus we can assume that $\mathfrak{s}^{c}$ contains $f, h, e$. We also call such an $\mathfrak{s}^{c}$ a carrier algebra of the triple $(f, h, e)$; note that $h / 2$ is its defining element.

Let $\mathfrak{h}_{0}^{c}$ be a fixed Cartan subalgebra of $\mathfrak{g}_{0}^{c}$. A carrier algebra $\mathfrak{s}^{c}$ is standard if it is normalized by $\mathfrak{h}_{0}^{c}$, and $\left[\mathfrak{h}_{0}^{c}, \mathfrak{s}_{k}\right] \subseteq \mathfrak{s}_{k}$ for all $k$. Since the Cartan subalgebras of $\mathfrak{g}_{0}^{c}$ are $G_{0}$-conjugate, every nilpotent $G_{0}$-orbit in $\mathfrak{g}_{1}^{c}$ corresponds to at least one standard carrier algebra $\mathfrak{s}^{c}$. Now, as shown in [Vinberg 1979, p. 23], the defining element of $\mathfrak{s}^{c}$ lies in $\mathfrak{h}_{0}^{c}$, and $\mathfrak{h}_{0}^{c} \cap \mathfrak{s}_{0}$ is a Cartan subalgebra of $\mathfrak{s}^{c}$; let $\Phi_{\mathfrak{s}^{c}}$ be the corresponding root system of $\mathfrak{s}^{c}$. Clearly, the homogeneous components $\mathfrak{s}_{k}$ are sums of root spaces, which allows us to define the degree of $\alpha \in \Phi_{\mathfrak{s}^{c}}$ as $\operatorname{deg}(\alpha)=k$ if $\mathfrak{s}_{\alpha} \subseteq \mathfrak{s}_{k}$. If $\Delta_{\mathfrak{s}} c$ is a basis of simple roots such that $\operatorname{deg}(\alpha) \geq 0$ for all $\alpha \in \Delta_{\mathfrak{s}} c$, then in fact $\operatorname{deg}(\alpha) \in\{0,1\}$; see [Vinberg 1979, p. 29]. If $\operatorname{deg}(\alpha)=1$ for all $\alpha \in \Delta_{\mathfrak{s}}$, then $\mathfrak{s}^{c}$ is principal. In that case $\mathfrak{s}_{0}=\mathfrak{s}_{0} \cap \mathfrak{h}_{0}^{c}$ is a torus (in particular, abelian) and $\mathfrak{s}_{1}$ is spanned by $\mathfrak{s}_{\alpha}$ with $\alpha \in \Delta_{\mathfrak{s}^{c}}$.

\section{Chevalley systems}

Again, we consider $\mathfrak{g}=\mathfrak{k} \oplus \mathfrak{p}$ with Cartan involution $\theta$ and complexification $\mathfrak{g}^{c}$ with complex conjugation $\sigma$. We suppose that $\mathfrak{h}^{c}=\mathfrak{h} \oplus \imath \mathfrak{h}$ is a Cartan subalgebra of $\mathfrak{g}^{c}$, where $\mathfrak{h}$ is a Cartan subalgebra of $\mathfrak{g}$ with $\mathfrak{h}=(\mathfrak{h} \cap \mathfrak{k}) \oplus(\mathfrak{h} \cap \mathfrak{p})$; write $\mathfrak{h}_{0}=\mathfrak{h} \cap \mathfrak{k}$ and $\mathfrak{a}=\mathfrak{h} \cap \mathfrak{p}$. In this situation, $\mathfrak{h}$ is called standard and an adjoint $\operatorname{ad}(h)$ has only purely imaginary eigenvalues if $h \in \mathfrak{h}_{0}$, and only real ones if $h \in \mathfrak{a}$ (see [Rothschild 1972, p. 405] or [Onishchik 2004, Proposition 5.1(ii)]). This condition on $\mathfrak{h}$ is not a serious restriction since every Cartan subalgebra of $\mathfrak{g}$ is conjugate to a standard Cartan subalgebra; see [Rothschild 1972, Proposition 1.3]. We let $\Phi$ be the root system of $\mathfrak{g}^{c}$ with respect to $\mathfrak{h}^{c}$, with basis of simple roots $\Delta=\left\{\alpha_{1}, \ldots, \alpha_{l}\right\}$. Further we assume that we have a canonical generating set $\left\{h_{i}, x_{i}, y_{i} \mid i\right\}$ such that for every $i$ either $\theta\left(x_{i}\right)=\lambda_{i} x_{i}$, with $\lambda_{i}= \pm 1$, or $\theta\left(x_{i}\right)=x_{j}$ with $i \neq j$. We extend these canonical generators to a Chevalley basis $\left\{h_{i}, x_{\alpha} \mid i, \alpha\right\}$. If $\alpha \in \Phi$ is a root, then $\beta=\alpha \circ \theta$ is a root with $\theta\left(x_{\alpha}\right) \in \mathfrak{g}_{\beta}$ and $\theta\left(h_{\alpha}\right)=h_{\beta}$; hence, by our assumptions, $\Delta$ is stable under $\alpha \mapsto \alpha \circ \theta$. Let $\pi$ be the permutation of $\{1, \ldots, l\}$ defined by $\alpha_{i} \circ \theta=\alpha_{\pi(i)}$. We retain this notation throughout this section. 
Lemma 7. For every $\alpha \in \Phi$ the following hold.

(a) $\theta\left(x_{\alpha}\right)=\lambda_{\alpha} x_{\alpha \circ \theta}$ for some $\lambda_{\alpha} \in\{ \pm 1\}$, and $\lambda_{\alpha}=\lambda_{\alpha}^{-1}=\lambda_{-\alpha}=\lambda_{\alpha \circ \theta}$.

(b) $\sigma\left(x_{\alpha}\right)=r_{\alpha} x_{-\alpha \circ \theta}$ for some $r_{\alpha} \in \mathbb{R}$, and $r_{\alpha}^{-1}=r_{-\alpha}=r_{-\alpha \circ \theta}$.

(c) $\theta\left(h_{\alpha}\right)=h_{\alpha \circ \theta}$ and $\sigma\left(h_{\alpha}\right)=h_{-\alpha \circ \theta}=-h_{\alpha \circ \theta}$.

Proof. (a) We already know that $\lambda_{\alpha_{i}}=\lambda_{i} \in\{ \pm 1\}$ and now use induction: If $\lambda_{\alpha}, \lambda_{\beta} \in\{ \pm 1\}$, then $N_{\alpha, \beta} \theta\left(x_{\alpha+\beta}\right)=\lambda_{\alpha} \lambda_{\beta} N_{\alpha \circ \theta, \beta \circ \theta} x_{(\alpha+\beta) \circ \theta}$, and hence $\lambda_{\alpha+\beta}=$ $N_{\alpha, \beta}^{-1} N_{\alpha \circ \theta, \beta \circ \theta} \lambda_{\alpha} \lambda_{\beta} \in\{ \pm 1\}$ since $\left|N_{\alpha, \beta}\right|=\left|N_{\alpha \circ \theta, \beta \circ \theta}\right|$; the latter holds since $\left|N_{\alpha, \beta}\right|=r+1$ where $r$ is the largest integer with $\alpha-r \beta \in \Phi$; see [Humphreys 1978, Theorem 25.2]. Since $\theta$ is an involution, $\lambda_{\alpha \circ \theta}=\lambda_{\alpha}^{-1}$, and $\lambda_{-\alpha}=\lambda_{\alpha}^{-1}$ follows from $h_{\alpha \circ \theta}=\theta\left(h_{\alpha}\right)=\theta\left(\left[x_{\alpha}, x_{-\alpha}\right]\right)=\lambda_{\alpha} \lambda_{-\alpha} h_{\alpha \circ \theta}$.

(b+c) Let $\left\{k_{1}, \ldots, k_{l}\right\}$ be a basis of $\mathfrak{h}^{c}=\mathfrak{h}_{0}^{c} \oplus \mathfrak{a}^{c}$ such that $\left\{k_{1}, \ldots, k_{m}\right\}$ and $\left\{k_{m+1}, \ldots, k_{l}\right\}$ form bases of $\mathfrak{a}$ and $\mathfrak{h}_{0}$, respectively. If $i \in\{1, \ldots, m\}$, then $\left[k_{i}, \sigma\left(x_{\alpha}\right)\right]=\sigma\left(\left[k_{i}, x_{\alpha}\right]\right)=\sigma\left(\alpha\left(k_{i}\right) x_{\alpha}\right)=\alpha\left(k_{i}\right) \sigma\left(x_{\alpha}\right)$ as $\alpha\left(k_{i}\right)$ is real. Analogously, if $i \in\{m+1, \ldots, l\}$, then $\alpha\left(k_{i}\right)$ is purely imaginary and $\left[k_{i}, \sigma\left(x_{\alpha}\right)\right]=-\alpha\left(k_{i}\right) \sigma\left(x_{\alpha}\right)$. Hence $\sigma\left(x_{\alpha}\right)=r_{\alpha} x_{-\alpha \circ \theta}$ with $r_{\alpha} \in \mathbb{C}$. Note that $h_{-\beta}=-h_{\beta}$ for all $\beta \in \Phi$. Now it follows from $\left[\sigma\left(h_{\alpha}\right), \sigma\left(x_{\alpha}\right)\right]=2 \sigma\left(x_{\alpha}\right)$ that $-\alpha \circ \theta\left(\sigma\left(h_{\alpha}\right)\right)=2$; hence $\sigma\left(h_{\alpha}\right) \in\left[\mathfrak{g}_{-\alpha \circ \theta}, \mathfrak{g}_{\alpha \circ \theta}\right]$ implies that

$$
\sigma\left(h_{\alpha}\right)=h_{-\alpha \circ \theta}=-h_{\alpha \circ \theta} .
$$

Since $\sigma\left(h_{\alpha}\right)=r_{\alpha} r_{-\alpha}\left[x_{-\alpha \circ \theta}, x_{\alpha \circ \theta}\right]=-r_{\alpha} r_{-\alpha} h_{\alpha \circ \theta}$, this already proves that $r_{\alpha} r_{-\alpha}=$ 1 for all $\alpha \in \Phi$. On the other hand, $r_{\alpha} \overline{r_{-\alpha}}=1$ (with - denoting the complex conjugate in $\mathbb{C}$ ) follows from

$r_{\alpha} \lambda_{-\alpha \circ \theta} x_{-\alpha}=\theta\left(r_{\alpha} x_{-\alpha \circ \theta}\right)=\theta \circ \sigma\left(x_{\alpha}\right)=\sigma \circ \theta\left(x_{\alpha}\right)=\lambda_{\alpha} \sigma\left(x_{\alpha \circ \theta}\right)=\lambda_{\alpha} \bar{r}_{-\alpha}^{-1} x_{-\alpha} ;$

recall that $\sigma \circ \theta=\theta \circ \sigma$ and $\lambda_{-\alpha \circ \theta}=\lambda_{\alpha}$ by (a). Together, we have $r_{\alpha} \in \mathbb{R}$ for all $\alpha \in \Phi$. Since $\sigma$ has order two, $r_{-\alpha \circ \theta}=r_{\alpha}^{-1}=r_{-\alpha}$ for all $\alpha \in \Phi$.

As for $\lambda_{i}=\lambda_{\alpha_{i}}$, we sometimes write $r_{i}=r_{\alpha_{i}}$. We now consider Chevalley systems as defined in [Bourbaki 1975, Chapter VIII, Section 3, Definition 3]; see also [Đoković 1987, Lemma 2].

Definition 8. We use the previous notation. A Chevalley system of $\mathfrak{g}^{c}$ with respect to $\mathfrak{h}^{c}$ is a family $\left(w_{\alpha}\right)_{\alpha \in \Phi}$ where $w_{\alpha} \in \mathfrak{g}_{\alpha}$ with $\left[w_{\alpha}, w_{-\alpha}\right]=-h_{\alpha}$ for all $\alpha \in \Phi$ and such that the linear map defined by $h \mapsto-h$ for $h \in \mathfrak{h}^{c}$ and $w_{\alpha} \mapsto w_{-\alpha}$ for $\alpha \in \Phi$ is a Lie automorphism, called Chevalley automorphism. If $\theta\left(w_{\alpha}\right)=\lambda_{\alpha} w_{\alpha \circ \theta}$ and $\sigma\left(w_{\alpha}\right)=\lambda_{\alpha} w_{-\alpha \circ \theta}$ for all $\alpha \in \Phi$ (with $\lambda_{\alpha}$ as in Lemma 7), then $\left(w_{\alpha}\right)_{\alpha \in \Phi}$ is called adapted with respect to $\mathfrak{g}=\mathfrak{k} \oplus \mathfrak{p}$ (and the Chevalley basis $\left\{h_{i}, x_{\alpha} \mid i, \alpha\right\}$ ).

We first show that adapted Chevalley systems exist. Then, for real forms of inner type, we construct an adapted Chevalley system from our given Chevalley basis; see [Đoković 1987, Lemma 2]. 
Lemma 9. There is an adapted Chevalley system $\left(v_{\alpha}\right)_{\alpha \in \Phi}$ of $\mathfrak{g}^{c}$ with respect to $\mathfrak{g}=\mathfrak{k} \oplus \mathfrak{p}$ and $\mathfrak{h}^{c}$.

Proof. For $\alpha \in \Phi$ let $z_{\alpha}=\varepsilon_{\alpha} x_{\alpha}$ where $\varepsilon_{\alpha}=-1$ if $\alpha \in \Phi^{-}$is negative, and $\varepsilon_{\alpha}=1$ otherwise. We first prove that $\left(z_{\alpha}\right)_{\alpha \in \Phi}$ is a Chevalley system of $\mathfrak{g}^{c}$. Clearly, $\left[z_{\alpha}, z_{-\alpha}\right]=$ $\varepsilon_{\alpha} \varepsilon_{-\alpha} h_{\alpha}=-h_{\alpha}$. Let $\psi$ be the linear map defined by $\psi(h)=-h$ for $h \in \mathfrak{h}^{c}$ and $\psi\left(z_{\alpha}\right)=z_{-\alpha}$ for $\alpha \in \Phi$. If $\alpha \in \Phi$, then $\varepsilon_{-\alpha}=-\varepsilon_{\alpha}$ and $\psi\left(x_{\alpha}\right)=\psi\left(\varepsilon_{\alpha} z_{\alpha}\right)=\varepsilon_{\alpha} z_{-\alpha}=$ $-x_{-\alpha}$. If $\alpha, \beta \in \Phi$ with $\alpha+\beta \in \Phi$, then $\psi\left(\left[z_{\alpha}, z_{\beta}\right]\right)=\psi\left(\varepsilon_{\alpha} \varepsilon_{\beta} N_{\alpha, \beta} x_{\alpha+\beta}\right)=$ $\varepsilon_{\alpha} \varepsilon_{\beta} \varepsilon_{\alpha+\beta} N_{\alpha, \beta} z_{-\alpha-\beta}$, and $N_{\alpha, \beta}=-N_{-\alpha,-\beta}$ yields $\psi\left(\left[z_{\alpha}, z_{\beta}\right]\right)=\left[\psi\left(z_{\alpha}\right), \psi\left(z_{\beta}\right)\right]$. Also, $\psi\left(\left[z_{\alpha}, z_{-\alpha}\right]\right)=\left[\psi\left(z_{\alpha}\right), \psi\left(z_{-\alpha}\right)\right]$ and $\psi\left(\left[h, z_{\alpha}\right]\right)=\left[\psi(h), \psi\left(z_{\alpha}\right)\right]$; thus $\psi$ is an automorphism and $\left(z_{\alpha}\right)_{\alpha \in \Phi}$ is a Chevalley system with respect to $\mathfrak{h}^{c}$.

We have seen in Section 2.2 that

$$
\tilde{\mathfrak{u}}=\operatorname{Span}_{\mathbb{R}}\left(\left\{l h_{1}, \ldots, l h_{l}, x_{\alpha}-x_{-\alpha}, l\left(x_{\alpha}+x_{-\alpha}\right) \mid \alpha \in \Phi^{+}\right\}\right)
$$

is a compact real form of $\mathfrak{g}^{c}$. If $\tilde{\tau}$ is the corresponding complex conjugation, then $\tilde{\tau}\left(x_{\alpha}\right)=-x_{-\alpha}$ for all $\alpha \in \Phi$ and $\tilde{\tau}\left(h_{i}\right)=-h_{i}$ for all $i$. In particular, $\tilde{\tau}$ and $\theta$ commute, and $\tilde{\sigma}=\theta \circ \tilde{\tau}$ is a real structure defining a real form $\tilde{\mathfrak{g}}=\mathfrak{g}(\theta, \tilde{\mathfrak{u}})=\tilde{\mathfrak{k}} \oplus \tilde{\mathfrak{p}}$ with Cartan involution $\theta$ (or, more precisely, the restriction of $\theta$ to $\tilde{\mathfrak{g}}$ ). If $\pi(i)=i$, then $l h_{i} \in \tilde{\mathfrak{k}}$; otherwise $l\left(h_{i}+h_{\pi(i)}\right) \in \tilde{\mathfrak{k}}$ and $h_{i}-h_{\pi(i)} \in \tilde{\mathfrak{p}}$ (see Section 2.2); thus $\tilde{\mathfrak{g}}$ has a standard Cartan subalgebra $\tilde{\mathfrak{h}}$ with $(\tilde{\mathfrak{h}})^{c}=\mathfrak{h}^{c}$. It follows readily from the definition of $\tilde{\sigma}$ that $\tilde{\sigma}\left(z_{\alpha}\right)=\lambda_{\alpha} z_{-\alpha \circ \theta}$ for all $\alpha \in \Phi$. Clearly, $\theta\left(z_{\alpha}\right)=\lambda_{\alpha} z_{\alpha \circ \theta}$, which shows that $\left(z_{\alpha}\right)_{\alpha \in \Phi}$ is an adapted Chevalley system with respect to $\tilde{\mathfrak{g}}=\tilde{\mathfrak{k}} \oplus \tilde{\mathfrak{p}}$ and $\mathfrak{h}^{c}$.

Set $\mathfrak{u}=\mathfrak{k} \oplus \imath \mathfrak{p}$. Then $\mathfrak{u}$ is the compact form of $\mathfrak{g}^{c}$ associated with the real form $\mathfrak{g}=\mathfrak{k} \oplus \mathfrak{p}$ (cf. Section 2.2). Let $\tau: \mathfrak{g}^{c} \rightarrow \mathfrak{g}^{c}$ be the complex conjugation with respect to $\mathfrak{u}$; then $\sigma=\theta \circ \tau$, and $\theta$ and $\tau$ commute. Thus, $\mathfrak{g}$ and $\tilde{\mathfrak{g}}$ both are real forms defined by the automorphism $\theta$ and the compact real structures $\tau$ and $\tilde{\tau}$, respectively. Using Lemma 7 , we get $\tau\left(x_{i}\right)=r_{i} \lambda_{i} y_{i}$ and $\tau\left(y_{i}\right)=r_{i}^{-1} \lambda_{i} x_{i}$. Let $\eta: \mathfrak{g}^{c} \rightarrow \mathfrak{g}^{c}$ be the automorphism which maps $\left(h_{i}, x_{i}, y_{i}\right)$ to $\left(h_{i},\left|r_{i}\right|^{-1 / 2} x_{i},\left|r_{i}\right|^{1 / 2} y_{i}\right)$ for all $i$. A short calculation shows that the compact structures $\eta^{-1} \circ \tau \circ \eta$ and $\tilde{\tau}$ commute. As shown in [Onishchik 2004, Proposition 3.5], commuting compact structures are equal; hence $\tau \circ \eta=\eta \circ \tilde{\tau}$. Again, using Lemma 7, we see that $\theta \circ \eta=\eta \circ \theta$, whence also $\sigma \circ \eta=\eta \circ \tilde{\sigma}$. Now consider $\left(v_{\alpha}\right)_{\alpha \in \Phi}$ with $v_{\alpha}=\eta\left(z_{\alpha}\right)$. Clearly, this is a Chevalley system: First, $v_{\alpha} \in \mathfrak{g}_{\alpha}$ and $\left[v_{\alpha}, v_{-\alpha}\right]=\eta\left(-h_{\alpha}\right)=-h_{\alpha}$ for all $\alpha \in \Phi$. Second, if $\psi$ is the Chevalley automorphism corresponding to $\left(z_{\alpha}\right)_{\alpha \in \Phi}$, then $\eta \circ \psi \circ \eta^{-1}$ is the Chevalley automorphism corresponding to $\left(v_{\alpha}\right)_{\alpha \in \Phi}$. Also, for $\alpha \in \Phi$ we have $\sigma\left(v_{\alpha}\right)=\sigma \circ \eta\left(z_{\alpha}\right)=\eta \circ \tilde{\sigma}\left(z_{\alpha}\right)=\lambda_{\alpha} v_{-\alpha \circ \theta}$ and $\theta\left(v_{\alpha}\right)=\theta \circ \eta\left(z_{\alpha}\right)=$ $\eta \circ \theta\left(z_{\alpha}\right)=\lambda_{\alpha} v_{\alpha \circ \theta}$, so $\left(v_{\alpha}\right)_{\alpha \in \Phi}$ is adapted with respect to $\mathfrak{g}=\mathfrak{k} \oplus \mathfrak{p}$.

Proposition 10. We use the previous notation. For $\alpha \in \Phi$ let $z_{\alpha}=\varepsilon_{\alpha} x_{\alpha}$ where $\varepsilon_{\alpha}=-1$ if $\alpha \in \Phi^{-}$is negative, and $\varepsilon_{\alpha}=1$ otherwise. Since $\sigma\left(z_{\alpha_{i}}\right)=-r_{i} z_{-\alpha_{\pi(i)}}$ 
with $r_{i}=r_{\pi(i)}$ by Lemma 7 , there are $\tau_{i}=\tau_{\pi(i)} \in \mathbb{R}$ such that $\tau_{i} \tau_{\pi(i)} r_{i} \in\{ \pm 1\}$. Let $\psi$ be the automorphism of $\mathfrak{g}^{c}$ mapping $\left(h_{i}, x_{i}, y_{i}\right)$ to $\left(h_{i}, \tau_{i} x_{i}, \tau_{i}^{-1} y_{i}\right)$ for all $i$; then $\psi$ commutes with $\theta$. Define $w_{\alpha}=\psi\left(z_{\alpha}\right)$ for $\alpha \in \Phi$.

(a) $\left(z_{\alpha}\right)_{\alpha \in \Phi}$ and $\left(w_{\alpha}\right)_{\alpha \in \Phi}$ are Chevalley systems with respect to $\mathfrak{h}^{c}$.

(b) $\theta\left(w_{\alpha_{i}}\right)=\lambda_{i} w_{\alpha_{\pi(i)}}$ and $\sigma\left(w_{\alpha_{i}}\right)=\lambda_{i} w_{-\alpha_{\pi(i)}}$ for all $i$.

(c) If $\mathfrak{g}$ is of inner type, then $\left(w_{\alpha}\right)_{\alpha \in \Phi}$ is adapted with respect to $\mathfrak{g}=\mathfrak{k} \oplus \mathfrak{p}$.

Proof. (a) This follows as in the proof of Lemma 9.

(b) Recall $z_{\alpha_{i}}=x_{i}$ and $z_{-\alpha_{\pi(i)}}=-y_{\pi(i)}$ for all $i$. Now $w_{-\alpha_{\pi(i)}}=\psi\left(z_{-\alpha_{\pi(i)}}\right)=$ $\tau_{\pi(i)}^{-1} z_{-\alpha_{\pi(i)}}$ yields

$\sigma\left(w_{\alpha_{i}}\right)=\sigma\left(\psi\left(z_{\alpha_{i}}\right)\right)=\sigma\left(\tau_{i} z_{\alpha_{i}}\right)=-\tau_{i} r_{i} z_{-\alpha_{\pi(i)}}=-\tau_{i} r_{i} \tau_{\pi(i)} w_{-\alpha_{\pi(i)}}=r_{i}^{\prime} w_{-\alpha_{\pi(i)}}$, where $r_{i}^{\prime}=-\tau_{i} \tau_{\pi(i)} r_{i} \in\{ \pm 1\}$. We have $\theta\left(x_{i}\right)=\lambda_{i} x_{i}$ if $\pi(i)=i$, and $\theta\left(x_{i}\right)=x_{\pi(i)}$ otherwise, and, therefore, $\tau_{i}=\tau_{\pi(i)}$ implies that $\theta\left(w_{\alpha_{i}}\right)=\lambda_{i} w_{\alpha_{i}}$ if $\pi(i)=i$, and $\theta\left(w_{\alpha_{i}}\right)=w_{\alpha_{\pi(i)}}$ otherwise. By Lemma 9, there exists an adapted Chevalley system $\left(v_{\alpha}\right)_{\alpha \in \Phi}$ with respect to $\mathfrak{g}=\mathfrak{k} \oplus \mathfrak{p}$ and $\mathfrak{h}^{c}$; each $v_{\alpha}$ can be written as $v_{\alpha}=c_{\alpha} w_{\alpha}$ for some $c_{\alpha} \in \mathbb{C}$. It follows from

$$
-h_{i}=\left[v_{\alpha_{i}}, v_{-\alpha_{i}}\right]=c_{\alpha_{i}} c_{-\alpha_{i}}\left[w_{\alpha_{i}}, w_{-\alpha_{i}}\right]=-c_{\alpha_{i}} c_{-\alpha_{i}} h_{i}
$$

that $c_{-\alpha_{i}}=c_{\alpha_{i}}^{-1}$ for all $i$. If $\pi(i) \neq i$, then

$$
c_{\alpha_{i}} w_{\alpha_{\pi(i)}}=\theta\left(c_{\alpha_{i}} w_{\alpha_{i}}\right)=\theta\left(v_{\alpha_{i}}\right)=v_{\alpha_{\pi(i)}}=c_{\alpha_{\pi(i)}} w_{\alpha_{\pi(i)}}
$$

hence $c_{\alpha_{i}}=c_{\alpha_{\pi(i)}}$ for all $i$. Thus $\overline{c_{\alpha_{i}}} c_{\alpha_{\pi(i)}}>0$ is real for every $i$, and $r_{i}^{\prime}=\lambda_{i}$ follows from $r_{i}^{\prime}, \lambda_{i} \in\{ \pm 1\}$ and

$$
\lambda_{i} v_{-\alpha_{\pi(i)}}=\sigma\left(v_{\alpha_{i}}\right)=\overline{c_{\alpha_{i}}} \sigma\left(w_{\alpha_{i}}\right)=\overline{c_{\alpha_{i}}} r_{i}^{\prime} c_{-\alpha_{\pi(i)}}^{-1} v_{-\alpha_{\pi(i)}}=r_{i}^{\prime} \overline{c_{\alpha_{i}}} c_{\alpha_{\pi(i)}} v_{-\alpha_{\pi(i)}} .
$$

(c) By (b) we know that $\sigma\left(w_{\alpha_{i}}\right)=\lambda_{\alpha_{i}} w_{-\alpha_{\pi(i)}}$, and Lemma 7 yields $\sigma\left(w_{-\alpha_{i}}\right)=$ $\lambda_{-\alpha_{i}} w_{\alpha_{\pi(i)}}$ for $i=1, \ldots, l$. For $\alpha, \beta \in \Phi$ with $\alpha+\beta \in \Phi$ write $\left[w_{\alpha}, w_{\beta}\right]=$ $M_{\alpha, \beta} w_{\alpha+\beta}$ where $M_{\alpha, \beta}=M_{-\alpha,-\beta}$ is real (in fact, integral). Suppose now that for $\alpha, \beta \in \Phi$ we have $\sigma\left(w_{\alpha}\right)=\lambda_{\alpha} w_{-\alpha \circ \theta}$ and $\sigma\left(w_{\beta}\right)=\lambda_{\beta} w_{-\beta \circ \theta}$. Then

$M_{\alpha, \beta} \sigma\left(w_{\alpha+\beta}\right)=\sigma\left(\left[w_{\alpha}, w_{\beta}\right]\right)=\left[\sigma\left(w_{\alpha}\right), \sigma\left(w_{\beta}\right)\right]=\lambda_{\alpha} \lambda_{\beta} M_{-\alpha \circ \theta,-\beta \circ \theta} w_{-(\alpha+\beta) \circ \theta}$.

If $\mathfrak{g}$ is of inner type, then $\alpha \circ \theta=\alpha$ and $\lambda_{\alpha} \lambda_{\beta}=\lambda_{\alpha+\beta}$ for all $\alpha, \beta \in \Phi$. Thus, in this case, $M_{\alpha, \beta}=M_{-\alpha \circ \theta,-\beta \circ \theta}=M_{-\alpha,-\beta}$, and induction on the height of $\alpha$ proves that $\sigma\left(w_{\alpha}\right)=\lambda_{\alpha} w_{-\alpha \circ \theta}$. Similarly, $\theta\left(w_{\alpha}\right)=\lambda_{\alpha} w_{\alpha \circ \theta}$ for all $\alpha$; thus $\left(w_{\alpha}\right)_{\alpha \in \Phi}$ is adapted with respect to $\mathfrak{g}=\mathfrak{k} \oplus \mathfrak{p}$.

The proof of Proposition 10(b) has the following important corollary, which we use in Section 7. Recall that $\theta\left(x_{i}\right)=\lambda_{i} x_{\pi(i)}$ and $\sigma\left(x_{i}\right)=r_{i} y_{\pi(i)}$ for all $i$.

Corollary 11. The coefficients $r_{i}$ and $-\lambda_{i}$ have the same sign for all $i$. 
Proof. In the proof of Proposition 10(b) we have shown that $\lambda_{i}=r_{i}^{\prime}=-\tau_{i} \tau_{\pi(i)} r_{i}=$ $-\tau_{i}^{2} r_{i}$.

\section{Constructing complex Cayley triples}

Let $\mathfrak{g}=\mathfrak{k} \oplus \mathfrak{p}$ be as in Section 2.2, with complexification $\mathfrak{g}^{c}$, Cartan involution $\theta$, and complex conjugation $\sigma$. As usual, we denote by $\Phi$ a root system of $\mathfrak{g}^{c}$ with basis of simple roots $\Delta$; let $\left\{h_{i}, x_{\alpha} \mid i, \alpha\right\}$ be a corresponding Chevalley basis. We now discuss our Main Problem (see Section 3); that is, given a homogeneous $\mathfrak{s l}_{2}$-triple $(f, h, e)$ in $\mathfrak{g}^{c}$, we want to construct a complex Cayley triple $\left(f^{\prime}, h^{\prime}, e^{\prime}\right)$ which is $K^{c}$-conjugate to $(f, h, e)$. As constructed in Section 4 , we also assume we have a standard carrier algebra $\mathfrak{s}^{c}=\mathfrak{s}^{c}(e, \mathfrak{t})$ containing $f, h, e$ (see Remark 6) and normalized by the Cartan subalgebra $\mathfrak{h}_{0}^{c}=\mathfrak{h}_{0}+\imath \mathfrak{h}_{0}$ of $\mathfrak{k}^{c}$ with $\mathfrak{h}_{0} \subseteq \mathfrak{k}$ as in Section 2.2.

We will see in Section 6.1 that $\mathfrak{s}^{c}$ is $\sigma$-stable; hence $\mathfrak{s}=\mathfrak{s}^{c} \cap \mathfrak{g}$ is a real form of $\mathfrak{s}^{c}$. Also, we will see that $\mathfrak{s}^{c}$ is $\theta$-stable; thus

$$
\mathfrak{s}=\left(\mathfrak{s}^{c} \cap \mathfrak{k}\right) \oplus\left(\mathfrak{s}^{c} \cap \mathfrak{p}\right)
$$

is a Cartan decomposition whose Cartan involution is the restriction of $\theta$ to $\mathfrak{s}$. Note that $\mathfrak{s}_{0} \cap \mathfrak{k}^{c}$ and $\mathfrak{s}_{0} \cap \mathfrak{k}$ contain Cartan subalgebras of $\mathfrak{s}^{c}$ and $\mathfrak{s}$, respectively, namely, $\mathfrak{h}_{0}^{c} \cap \mathfrak{s}_{0}$ and $\mathfrak{h}_{0} \cap \mathfrak{s}_{0}$. In particular, the real form $\mathfrak{s}$ is always of inner type and $\mathfrak{h}_{0} \cap \mathfrak{s}_{0}$ is a standard Cartan subalgebra. Thus the results of Section 5 can be applied: we show in Section 6.1 how to construct an adapted Chevalley system for $\mathfrak{s}^{c}$; here adapted always means with respect to $\mathfrak{h}_{0}^{c} \cap \mathfrak{s}_{0}$, the Cartan decomposition ( $\star$ ), and a chosen Chevalley basis of $\mathfrak{s}^{c}$.

By construction, the triple $(f, h, e)$ is also a homogeneous $\mathfrak{s l}_{2}$-triple in $\mathfrak{s}^{c}$. The approach of [Đoković 1987] is to find $x \in \mathfrak{s}_{1}$ with $[x, \sigma(x)]=h$ so that $(\sigma(x), h, x)$ is a complex Cayley triple in $\mathfrak{s}^{c}$, thus also in $\mathfrak{g}^{c}$. By the Kostant-Sekiguchi correspondence and [Kostant and Rallis 1971, Lemma 4], such an $x$ exists and $(\sigma(x), h, x)$ is $K^{c}$-conjugate to $(f, h, e)$. If $\mathfrak{s}^{c}$ is principal, then Chevalley systems can be used to find $x$; see Section 6.2. If $\mathfrak{s}^{c}$ is not principal, then we make a case distinction and use induction; see Section 6.3.

6.1. Constructing an adapted Chevalley system. In the following, let $\Phi_{\mathfrak{s}^{c}}$ be the root system of $\mathfrak{s}^{c}$ with respect to $\mathfrak{h}_{0}^{c} \cap \mathfrak{s}_{0}$; let $\Delta_{\mathfrak{s}^{c}}=\left\{\beta_{1}, \ldots, \beta_{s}\right\}$ be a basis of simple roots. As mentioned in Section 4 , we can assume that each root space $\mathfrak{s}_{\beta_{i}}$ either lies in $\mathfrak{s}_{0}$ or $\mathfrak{s}_{1}$.

6.1.1. Inner type. If $\mathfrak{g}$ is of inner type, then $\mathfrak{h}_{0}^{c} \leq \mathfrak{k}^{c}$ is also a Cartan subalgebra of $\mathfrak{g}^{c}$; hence $\Phi_{\mathfrak{s}^{c}}$ can be considered as a root subsystem of $\Phi$. This implies that $\left\{x_{\alpha} \mid \alpha \in \Phi_{\mathfrak{s}^{c}}\right\}$, along with certain elements of $\mathfrak{h}_{0}^{c} \cap \mathfrak{s}_{0}$, forms a Chevalley basis 
of $\mathfrak{s}^{c}$. We denote it by $\left\{k_{i}, w_{\alpha} \mid \alpha \in \Phi_{\mathfrak{s}^{c}}, i=1, \ldots, s\right\}$ and let $\left\{k_{i}, a_{i}, b_{i} \mid i\right\}$ be the canonical generating set it contains. As usual, write $k_{\alpha}=\left[w_{\alpha}, w_{-\alpha}\right]$ for $\alpha \in \Phi_{\mathfrak{s}^{c}}$. We have seen in Section 2.2.2 that $\sigma\left(w_{\alpha}\right) \in\left\{ \pm w_{-\alpha}\right\}$ and $\theta\left(w_{\alpha}\right) \in\left\{ \pm w_{\alpha}\right\}$; hence $\mathfrak{s}^{c}$ is $\sigma$ - and $\theta$-stable. For $\Phi_{\mathfrak{s}^{c}}$ we use an ordering compatible with that of $\Phi$. Let $z_{\alpha}=w_{\alpha}$ and $z_{-\alpha}=-w_{-\alpha}$ for $\alpha \in \Phi_{\mathfrak{s} c}^{+}$; then $\left(z_{\alpha}\right)_{\alpha \in \Phi_{\mathfrak{s} c} c}$ is an adapted Chevalley system of $\mathfrak{s}^{c}$.

6.1.2. Outer type. Now let $\mathfrak{g}$ be of outer type with defining outer automorphism $\theta=\varphi \circ \chi$. By construction, each homogeneous component $\mathfrak{s}_{k}$ lies either in $\mathfrak{k}^{c}$ or in $\mathfrak{p}^{c}$, which shows that $\mathfrak{s}^{c}$ is $\theta$-stable. By definition, each $\mathfrak{s}_{k}$ is normalized by $\mathfrak{h}_{0}^{c}$; thus it is a sum of weight spaces (with respect to $\mathfrak{h}_{0}^{c}$ ) as considered in Sections 2.2.3 and 2.2.4; in the following we use the notation introduced in these sections. Let $\alpha \in \Phi_{\mathfrak{s}^{c}}$; then $\mathfrak{s}_{\alpha}$ is an $\mathfrak{h}_{0}^{c}$-weight space, and it is either contained in $\mathfrak{k}^{c}$ or in $\mathfrak{p}^{c}$ (since it lies in a homogeneous component $\mathfrak{s}_{k}$ ). These observations show that there is an $\alpha^{\prime} \in \Phi$ such that either $\mathfrak{s}_{\alpha}=\operatorname{Span}_{\mathbb{C}}\left(u_{\alpha^{\prime}}\right)$ or $\mathfrak{s}_{\alpha}=\operatorname{Span}_{\mathbb{C}}\left(v_{\alpha^{\prime}}\right)$, and, accordingly, $\mathfrak{s}_{-\alpha}=\operatorname{Span}_{\mathbb{C}}\left(u_{-\alpha^{\prime}}\right)$ or $\mathfrak{s}_{-\alpha}=\operatorname{Span}_{\mathbb{C}}\left(v_{-\alpha^{\prime}}\right)$. Since $\sigma\left(u_{\alpha^{\prime}}\right)= \pm u_{-\alpha^{\prime}}$ and $\sigma\left(v_{\alpha}\right)= \pm v_{-\alpha^{\prime}}$, this shows that $\mathfrak{s}^{c}$ is stable under $\sigma$. We can now define a new set of canonical generators $\left\{k_{i}, a_{i}, b_{i} \mid i=1, \ldots, s\right\}$ for $\mathfrak{s}$; we make a case distinction:

- If $\mathfrak{s}_{i}$ is spanned by $u_{\alpha}=x_{\alpha}$ with $\varphi(\alpha)=\alpha$, then define $a_{i}=x_{\alpha}, b_{i}=x_{-\alpha}$ and $k_{i}=\left[a_{i}, b_{i}\right]$.

- Now let $\mathfrak{s}_{i}$ be spanned by $u_{\alpha}=x_{\alpha}+x_{\varphi(\alpha)}$ with $\varphi(\alpha) \neq \alpha$. Note that $\beta=\alpha-\varphi(\alpha)$ is not a root because $\varphi$ maps positive roots on positive roots but $\varphi(\beta)=-\beta$. This proves $\left[u_{\alpha}, u_{-\alpha}\right]=h_{\alpha}+h_{\varphi(\alpha)}$. Also, it follows that $\langle\alpha, \varphi(\alpha)\rangle \leq 0$ (see [Humphreys 1978, Lemma 9.4]) and finally $\langle\alpha, \varphi(\alpha)\rangle \in$ $\{0,-1\}$, as $\Phi$ is simply laced, which means that there is only one root length; in particular, $\langle\alpha, \varphi(\alpha)\rangle=\langle\varphi(\alpha), \alpha\rangle$. The latter now implies that $\left[h_{\alpha}+h_{\varphi(\alpha)}, u_{\alpha}\right]=$ $(2+\langle\varphi(\alpha), \alpha\rangle) u_{\alpha}$ since $\varphi(\alpha)\left(h_{\alpha}\right)=\langle\varphi(\alpha), \alpha\rangle=\langle\alpha, \varphi(\alpha)\rangle=\alpha\left(h_{\varphi(\alpha)}\right)$. If $\langle\varphi(\alpha), \alpha\rangle=0$, then we define $a_{i}=u_{\alpha}, b_{i}=u_{-\alpha}$, and $k_{i}=\left[a_{i}, b_{i}\right]$. Otherwise, we set $a_{i}=\sqrt{2} u_{\alpha}, b_{i}=\sqrt{2} u_{-\alpha}$, and $k_{i}=\left[a_{i}, b_{i}\right]$.

- If $\mathfrak{s}_{i}$ is spanned by $v_{\alpha}=x_{\alpha}-x_{\varphi(\alpha)}$, then we do exactly the same as in the previous case with $u$ replaced by $v$.

In all cases we find $a_{i} \in \mathfrak{s}_{\beta_{i}}, b_{i} \in \mathfrak{s}_{-} \beta_{i}$, and $k_{i}=\left[a_{i}, b_{i}\right]$ such that $\left[k_{i}, a_{i}\right]=2 b_{i}$ for all $i$. By Proposition $3,\left\{k_{i}, a_{i}, b_{i} \mid i\right\}$ is a canonical generating set for $\mathfrak{s}^{c}$, and, by construction, $\sigma\left(a_{i}\right)= \pm b_{i}$ for all $i$. We extend this canonical generating set to a Chevalley basis $\left\{k_{i}, w_{\alpha} \mid i, \alpha\right\}$ of $\mathfrak{s}^{c}$ such that $w_{\alpha_{i}}=a_{i}$ and $w_{-\alpha_{i}}=b_{i}$; as usual, write $k_{\alpha}=\left[w_{\alpha}, w_{-\alpha}\right]$ for all $\alpha$. We now define $z_{\alpha}=w_{\alpha}$ for $\alpha>0$ and $z_{\alpha}=-w_{\alpha}$ for $\alpha<0$; it is straightforward to verify that $\left(z_{\alpha}\right)_{\alpha \in \Phi_{\mathrm{s} c}}$ is an adapted Chevalley system of $\mathfrak{s}^{c}$; see Proposition 10. 
The conclusion is that for all $\mathfrak{g}$ we can find an adapted Chevalley system of $\mathfrak{s}^{c}$, and the coefficients of its elements with respect to the given basis of $\mathfrak{g}$ lie in $\mathbb{Q}(l, \sqrt{2})$; in particular, in $\mathbb{Q} \sqrt{ }(l)$.

6.2. The principal case. This construction follows [Đoković 1987, Lemma 3]. We use the previous notation and suppose that the carrier algebra $\mathfrak{s}^{c}$ of $(f, h, e)$ is principal; that is, there is a basis $\Delta_{\mathfrak{s}^{c}}$ of $\Phi_{\mathfrak{s}^{c}}$ such that for every $\alpha \in \Delta_{\mathfrak{s}^{c}}$ we have $\mathfrak{s}_{\alpha} \subseteq \mathfrak{s}_{1}$. Let $\left(z_{\alpha}\right)_{\alpha \in \Phi_{\mathfrak{s}} c}$ be the adapted Chevalley system for $\mathfrak{s}^{c}$ as constructed in the previous section. We want to find $x \in \mathfrak{s}_{1}$ with $[x, \sigma(x)]=h$ of the form $x=\sum_{\alpha \in \Delta_{s} c} c_{\alpha} z_{\alpha}$ with all $c_{\alpha}$ real. Note that $\sigma(x)=-\sum_{\alpha \in \Delta_{\mathfrak{s}} c} c_{\alpha} z_{-\alpha}$ and $\alpha-\beta \notin \Phi_{\mathfrak{s} c}$ for all $\alpha, \beta \in \Delta_{\mathfrak{s} c}$. Thus, the equation we have to solve is $h=[x, \sigma(x)]=\sum_{\alpha \in \Delta_{\mathfrak{s}} c} c_{\alpha}^{2} k_{\alpha}$; recall that $\left[z_{\alpha}, z_{-\alpha}\right]=-k_{\alpha}$. Note that $\beta(h)=2$ for all $\beta \in \Delta_{\mathfrak{s}} c$ since $z_{\beta} \in \mathfrak{s}_{1}$ and $h / 2$ is the defining element of $\mathfrak{s}^{c}$. This shows that our equation is equivalent to the system of equations $2=\sum_{\alpha \in \Delta_{\mathfrak{s}} c} d_{\alpha} \beta\left(k_{\alpha}\right)$ with $d_{\alpha}=c_{\alpha}^{2}$ where $\beta$ ranges over $\Delta_{\mathfrak{s}}$. The coefficients $\beta\left(k_{\alpha}\right)$ of this system are the entries of the Cartan matrix of $\Phi_{\mathfrak{s}^{c}}$, whose inverse has nonnegative entries; see [Humphreys 1978, Section 13.1]. Thus, the system has a solution with all $d_{\alpha} \geq 0$ real. In conclusion, to construct $x$, we first compute $h=\sum_{\alpha \in \Delta_{\mathfrak{s}} c} d_{\alpha} k_{\alpha}$, and then set $x=\sum_{\alpha \in \Delta_{\mathfrak{s}} c} c_{\alpha} z_{\alpha}$ where $c_{\alpha}=\sqrt{d_{\alpha}}$ is real for every $\alpha \in \Delta_{\mathfrak{s}^{c}}$.

We now show that each $c_{\alpha} \in \mathbb{Q} \sqrt{ }$. For every $\alpha \in \Delta_{\mathfrak{s}^{c}} \subseteq \Phi_{\mathfrak{s}^{c}}$, the element $k_{\alpha}=\left[w_{\alpha}, w_{-\alpha}\right]$ is a $\mathbb{Z}$-linear combination of $k_{1}, \ldots, k_{s}$, the elements of the Chevalley basis of $\mathfrak{s}^{c}$ that span its Cartan subalgebra $\mathfrak{h}_{0}^{c} \cap \mathfrak{s}_{0}$; see [Humphreys 1978, Theorem 25.2]. As shown in the previous paragraph, these elements are $\mathbb{Z}$-linear combinations of $h_{1}, \ldots, h_{l}$, the elements of the Chevalley basis of $\mathfrak{g}^{c}$ that span $\mathfrak{h}^{c}$. Similarly, the element $h$, which is the characteristic of an $\mathfrak{s l}_{2}$-triple, is a $\mathbb{Z}$-linear combination of $h_{1}, \ldots, h_{l}$. Together, all this implies that the $d_{\alpha}$ are in fact rational; thus $c_{\alpha} \in \mathbb{Q} \sqrt{ }$.

6.3. Nonprincipal case. Now suppose that the carrier algebra $\mathfrak{s}^{c}$ of $(f, h, e)$ is nonprincipal. As mentioned above, there exists $x \in \mathfrak{s}_{1}$ such that $(\sigma(x), h, x)$ is a complex Cayley triple in the same $K^{c}$-orbit as $(f, h, e)$. However, constructing $x$ is not straightforward. We first set up the system of rational polynomial equations in the coefficients of $x$ with respect to a basis of $\mathfrak{s}_{1}$, equivalent to $[x, \sigma(x)]=h$. Note that this is a system of $\operatorname{dim} \mathfrak{s}_{0}$ polynomial equations in $\operatorname{dim} \mathfrak{s}_{1}$ variables. Then in order to solve them we use a brute-force approach; that is, for $i=1,2,3, \ldots$, we set all but $i$ indeterminates in these equations to zero. For each equation system that arises we check, using Gröbner bases (see for example [Cox et al. 1992]), whether a solution over $\mathbb{C}$ exists. We stop when we find an equation system consisting of equations of the form $T^{2}=a$, where $a \in \mathbb{Q}$ and $T$ is an indeterminate, or $T_{c}=a_{c_{1}} T_{c_{1}}^{2}+\cdots+a_{c_{m}} T_{c_{m}}^{2}$, where each $T_{c_{i}}$ satisfies an equation of the first type. It is then straightforward to obtain a solution over $\mathbb{Q} \sqrt{ }(l)$. This systematic approach 
for constructing a complex Cayley triple $(\sigma(x), h, x)$ can easily be carried out automatically by a computer. It turned out to work well in all our computations for the carrier algebras in the real forms constructed in Section 2.2; our experiments include all simple real Lie algebras of rank at most 8 . Unfortunately, we have no proof that a solution of the equation system always exists over the field $\mathbb{Q} \sqrt{ }(l)$; hence we cannot prove that our approach will always work.

6.3.1. A database. To reduce work, we have constructed a database of the simple nonprincipal carrier algebras that appeared during our calculations. Let $\mathfrak{s}^{c}$ be such a carrier algebra. As shown in Section 6.1, there is a canonical generating set $\left\{k_{i}, a_{i}, b_{i} \mid i=1, \ldots, s\right\}$ of $\mathfrak{s}^{c}$ such that $a_{i} \in \mathfrak{s}_{\varepsilon_{i}}$ with $\varepsilon_{i} \in\{0,1\}$ and $\sigma\left(a_{i}\right)=\lambda_{i} b_{i}$ with $\lambda_{i} \in\{ \pm 1\}$ for all $i$. Since $\sigma\left(k_{i}\right)=-k_{i}$ for all $i$, the map $\sigma$ is determined by the signs $\lambda_{1}, \ldots, \lambda_{s}$. Moreover, $k_{1}, \ldots, k_{s} \in \mathfrak{s}_{0}$, and, if $a_{i} \in \mathfrak{s}_{k}$, then $b_{i} \in \mathfrak{s}_{-k}$. Thus, the following data describes $\mathfrak{s}^{c}$, its grading, and $\sigma$ completely; we store this data in our database:

- a multiplication table, canonical generators $\left\{k_{i}, a_{i}, b_{i} \mid i\right\}$, and Cartan matrix $C$,

- the signs $\lambda_{1}, \ldots, \lambda_{s}$ and $\varepsilon_{1}, \ldots, \varepsilon_{s}$,

- a complex Cayley triple $(f, h, e)$ in $\mathfrak{s}$ such that $e \in \mathfrak{s}_{1}$ is in general position.

Suppose in our computations we consider a real semisimple Lie algebra $\mathfrak{g}^{\prime}=\mathfrak{k}^{\prime} \oplus \mathfrak{p}^{\prime}$ with complexification $\left(\mathfrak{g}^{\prime}\right)^{c}=\left(\mathfrak{k}^{\prime}\right)^{c} \oplus\left(\mathfrak{p}^{\prime}\right)^{c}$ and complex conjugation $\sigma^{\prime}$. Let $\left(f^{\prime}, h^{\prime}, e^{\prime}\right)$ be a homogeneous $\mathfrak{s l}_{2}$-triple in $\left(\mathfrak{g}^{\prime}\right)^{c}$, and we want to find a conjugate complex Cayley triple in $\left(\mathfrak{g}^{\prime}\right)^{c}$. As before, we first construct the carrier algebra $\left(\mathfrak{s}^{\prime}\right)^{c}$ of the triple. If it is principal, then we proceed as in Section 6.2, so let it be nonprincipal. Recall that $\left(\mathfrak{s}^{\prime}\right)^{c}$ is semisimple and, by considering its simple components separately, we can assume that $\left(\mathfrak{s}^{\prime}\right)^{c}$ itself is simple. Suppose in our database there exists a simple carrier algebra $\mathfrak{s}^{c}$ whose parameters as described above satisfy the following:

(1) $\left(\mathfrak{s}^{\prime}\right)^{c}$ has canonical generators $\left\{k_{i}^{\prime}, a_{i}^{\prime}, b_{i}^{\prime} \mid i\right\}$ with Cartan matrix $C$,

(2) if $\sigma^{\prime}\left(a_{i}^{\prime}\right)=\lambda_{i}^{\prime} b_{i}^{\prime}$, then $\operatorname{sgn}\left(\lambda_{i}^{\prime}\right)=\operatorname{sgn}\left(\lambda_{i}\right)$ for all $i$,

(3) if $a_{i}^{\prime} \in \mathfrak{s}_{\varepsilon_{i}^{\prime}}^{\prime}$, then $\varepsilon_{i}^{\prime}=\varepsilon_{i}$ for all $i$.

If all this holds, then we can get a complex Cayley triple in $\left(\mathfrak{s}^{\prime}\right)^{c}$ as follows. Let $\varphi$ be the isomorphism from $\mathfrak{s}^{c}$ to $\left(\mathfrak{s}^{\prime}\right)^{c}$ which maps $\left(k_{i}, a_{i}, b_{i}\right)$ to $\left(k_{i}^{\prime}, \mu_{i} a_{i}^{\prime}, \mu_{i}^{-1} b_{i}^{\prime}\right)$, where $\mu_{i}=\sqrt{\lambda_{i} / \lambda_{i}^{\prime}}$ for all $i$. Obviously $\varphi$ is an isomorphism of $\mathbb{Z}$-graded Lie algebras. A short calculation shows that the antilinear homomorphisms $\varphi \circ \sigma$ and $\sigma^{\prime} \circ \varphi$ agree on the canonical generators of $\mathfrak{s}^{c}$; thus $\varphi \circ \sigma=\sigma^{\prime} \circ \varphi$. Since $\varphi$ maps the unique defining element $h / 2$ of $\mathfrak{s}^{c}$ onto the unique defining element $h^{\prime} / 2$ of $\left(\mathfrak{s}^{\prime}\right)^{c}$, we have $h^{\prime}=\varphi(h)$. Let $x=\varphi(e)$ and $y=\varphi(f)$; then $\left(y, h^{\prime}, x\right)$ is a complex Cayley triple in $\left(\mathfrak{s}^{\prime}\right)^{c}$. Since $x \in \mathfrak{s}_{1}^{\prime}$ is in general position, $\left(y, h^{\prime}, x\right)$ is $\left(K^{\prime}\right)^{c}$-conjugate 
to $\left(f^{\prime}, h^{\prime}, e^{\prime}\right)$. The conclusion is that by storing the simple carrier algebras in a database we can find a complex Cayley triple in a carrier algebra by a look-up in the database.

\section{Isomorphisms}

Let $\mathfrak{g}^{c}$ be a simple complex Lie algebra with real form $\mathfrak{g}=\mathfrak{k} \oplus \mathfrak{p}$ and Cartan involution $\theta$ and complex conjugation $\sigma$. As usual, we extend $\theta$ to an automorphism of $\mathfrak{g}^{c}$. Let $\left(\mathfrak{g}^{\prime}\right)^{c}$ be a second simple complex Lie algebra with real form $\mathfrak{g}^{\prime}=\mathfrak{k}^{\prime} \oplus \mathfrak{p}^{\prime}$, Cartan involution $\theta^{\prime}$, and complex conjugation $\sigma^{\prime}$. We consider the problem to decide whether $\mathfrak{g}$ and $\mathfrak{g}^{\prime}$ are isomorphic, and, if they are, to find an isomorphism. For this we may obviously assume that $\mathfrak{g}^{c}$ and $\left(\mathfrak{g}^{\prime}\right)^{c}$ are isomorphic.

Recall that a Cartan decomposition is unique up to conjugacy; see [Onishchik 2004, Theorem 5.1]. Thus, if $\mathfrak{g}$ and $\mathfrak{g}^{\prime}$ are isomorphic, then there also exists an isomorphism $\psi: \mathfrak{g} \rightarrow \mathfrak{g}^{\prime}$ with $\psi(\mathfrak{k})=\mathfrak{k}^{\prime}$ and $\psi(\mathfrak{p})=\mathfrak{p}^{\prime}$. Clearly, such an isomorphism extends to an isomorphism $\psi: \mathfrak{g}^{c} \rightarrow\left(\mathfrak{g}^{\prime}\right)^{c}$ with $\psi \circ \theta=\theta^{\prime} \circ \psi$ and $\psi \circ \sigma=\sigma^{\prime} \circ \psi$. Conversely, if we find an isomorphism

$$
\psi: \mathfrak{g}^{c} \rightarrow\left(\mathfrak{g}^{\prime}\right)^{c} \quad \text { with } \psi \circ \theta=\theta^{\prime} \circ \psi \text { and } \psi \circ \sigma=\sigma^{\prime} \circ \psi,
$$

then $\psi$ restricts to an isomorphism $\psi: \mathfrak{g} \rightarrow \mathfrak{g}^{\prime}$ with $\psi(\mathfrak{k})=\mathfrak{k}^{\prime}$ and $\psi(\mathfrak{p})=\mathfrak{p}^{\prime}$.

We now describe a construction of the isomorphism $(*)$, which fails if and only if $\mathfrak{g}$ and $\mathfrak{g}^{\prime}$ are not isomorphic. Our main tool is the technique described in the following preliminary section.

7.1. Weyl group action. We consider the following setup. Let $\mathfrak{h}^{c} \leq \mathfrak{g}^{c}$ be a Cartan subalgebra of $\mathfrak{g}^{c}$ with corresponding root system $\Phi$ and basis of simple roots $\Delta=$ $\left\{\alpha_{1}, \ldots, \alpha_{l}\right\}$. Let $W$ be the Weyl group associated to $\Phi$. As usual, let $\left\{h_{i}, x_{i}, y_{i} \mid i\right\}$ be a canonical generating set contained in a Chevalley basis $\left\{h_{i}, x_{\alpha} \mid i, \alpha\right\}$ of $\mathfrak{g}^{c}$. Note that $\theta\left(x_{\alpha}\right) \in \mathfrak{g}_{\alpha \circ \theta}$, and we suppose that $\alpha \mapsto \alpha \circ \theta$ preserves $\Delta$. Then $\theta=\varphi \circ \chi=\chi \circ \varphi$, where $\varphi$ is a diagram automorphism permuting $\Delta$, and $\chi$ is an inner automorphism with $\chi(h)=h$ for all $h \in \mathfrak{h}^{c}$. Let the permutation $\pi$ be defined by $\varphi\left(\alpha_{i}\right)=\alpha_{\pi(i)}$. We further suppose that $\theta\left(x_{\alpha}\right)=\lambda_{\alpha} x_{\alpha \circ \theta}$ with $\lambda_{\alpha}=1$ if $\alpha \circ \theta \neq \alpha$. Thus, $\lambda_{\alpha} \in\{ \pm 1\}$ for all $\alpha \in \Phi$; we write $\lambda_{i}=\lambda_{\alpha_{i}}$ and call $\lambda_{1}, \ldots, \lambda_{l}$ the parameters of $\theta$.

By abuse of notation, to $w \in W$ we associate the automorphism $w \in \operatorname{Aut}\left(\mathfrak{g}^{c}\right)$ which maps $\left(h_{i}, x_{i}, y_{i}\right)$ to $\left(h_{w\left(\alpha_{i}\right)}, x_{w\left(\alpha_{i}\right)}, x_{-w\left(\alpha_{i}\right)}\right)$ for all $i$. Let $\Delta_{\theta}=\{\alpha \in \Delta \mid$ $\alpha \circ \theta=\alpha\}$, let $\Phi_{\theta}$ be the root subsystem of $\Phi$ with basis $\Delta_{\theta}$, and let $W_{\theta}$ be its Weyl group.

Lemma 12. If $w=s_{\alpha_{k}} \in W_{\theta}$, then $\alpha \rightarrow \alpha \circ \theta$ preserves the basis of simple roots $w(\Delta)$. 
Proof. This follows readily if $\theta$ is inner since then $\varphi$ is the identity and $\alpha \circ \theta=\alpha$ for all $\alpha \in \Phi$. So suppose $\varphi$ is not the identity; hence $\Phi$ is simply laced; see [Onishchik 2004, Table 1]. Note that $\pi(k)=k$; thus $\theta\left(w\left(x_{k}\right)\right)=\theta\left(y_{k}\right)=\lambda_{k} y_{k}=\lambda_{k} w\left(x_{k}\right)$, which shows $w\left(\alpha_{k}\right) \circ \theta=w\left(\alpha_{k}\right) \in w(\Delta)$. If $j$ is such that $\left\langle\alpha_{j}, \alpha_{k}\right\rangle=-1$, then $w\left(\alpha_{j}\right)=\alpha_{j}+\alpha_{k}$ and $w\left(x_{j}\right)=x_{\alpha_{k}+\alpha_{j}}$. Since $\Phi$ is simply laced, $N_{\alpha, \beta}= \pm 1$ for all $\alpha, \beta \in \Phi$, and $\left[x_{k}, x_{j}\right]=N_{\alpha_{k}, \alpha_{j}} x_{\alpha_{k}+\alpha_{j}}$ implies that $\theta\left(w\left(x_{j}\right)\right)= \pm x_{\alpha_{k}+\alpha_{\pi(j)}}$. Since also $\left\langle\alpha_{\pi(j)}, \alpha_{k}\right\rangle=-1$, we have $x_{\alpha_{k}+\alpha_{\pi(j)}}=w\left(x_{\pi(j)}\right)$; hence $w\left(\alpha_{j}\right) \circ$ $\theta=w\left(\alpha_{\pi(j)}\right) \in w(\Delta)$. Analogously, if $\left\langle\alpha_{j}, \alpha_{k}\right\rangle=0$, then $w\left(x_{j}\right)=x_{j}$; hence $w\left(\alpha_{j}\right) \circ \theta=w\left(\alpha_{\pi(j)}\right) \in w(\Delta)$.

Suppose $\theta$ has parameters $\lambda_{1}, \ldots, \lambda_{l}$, that is, $\theta\left(x_{i}\right)=\lambda_{i} x_{\pi(i)}$ for all $i$, and let $w=s_{\alpha_{k}} \in W_{\theta}$. Clearly, $\left\{w\left(h_{i}\right), w\left(x_{i}\right), w\left(y_{i}\right) \mid i\right\}$ is a canonical generating set, and we modify it as follows: whenever $\pi(i)>i$, we replace $w\left(x_{\pi(i)}\right)$ and $w\left(y_{\pi(i)}\right)$ by $\theta\left(w\left(x_{i}\right)\right)$ and $\theta\left(w\left(y_{i}\right)\right)$; let $\left\{\bar{h}_{i}, \bar{x}_{i}, \bar{y}_{i} \mid i\right\}$ be the resulting canonical generating set with corresponding basis of simple roots $w(\Delta)$, which still is $\theta$-stable by Lemma 12 . By construction, if $\pi(i) \neq i$, then $\theta\left(\bar{x}_{i}\right)=\bar{x}_{\pi(i)}$. Now let $\pi(j)=j$ and recall that $w\left(\alpha_{j}\right)=\alpha_{j}-\left\langle\alpha_{j}, \alpha_{k}\right\rangle \alpha_{k}$ and $\pi(k)=k$. A case distinction on the value of $\left\langle\alpha_{j}, \alpha_{k}\right\rangle$ shows that

$$
\theta\left(w\left(x_{j}\right)\right)=\lambda_{j} \lambda_{k}^{\left\langle\alpha_{j}, \alpha_{k}\right\rangle} w\left(x_{j}\right) .
$$

In conclusion, if we replace our original canonical generators and basis of simple roots by their (modified) images under $w \in \operatorname{Aut}\left(\mathfrak{g}^{c}\right)$, then for the parameters $\tilde{\lambda}_{j}$ of $\theta$ we have $\tilde{\lambda}_{j}=1$ if $\pi(j) \neq j$ and $\tilde{\lambda}_{j}=\lambda_{j} \lambda_{k}^{\left\langle\alpha_{j}, \alpha_{k}\right\rangle}$ if $\pi(j)=j$.

7.2. Inner type. First we suppose that $\mathfrak{g}$ is of inner type; that is, $\mathfrak{k}$ contains a Cartan subalgebra $\mathfrak{h}$ of $\mathfrak{g}$. Let $\Phi$ be the root system of $\mathfrak{g}^{c}$ with respect to $\mathfrak{h}^{c}$, with basis of simple roots $\Delta=\left\{\alpha_{1}, \ldots, \alpha_{l}\right\}$. Let $\left\{h_{i}, x_{i}, y_{i} \mid i\right\}$ be a canonical generating set corresponding to $\Delta$. If $\mathfrak{g}^{\prime}$ is not of inner type, that is, if a Cartan subalgebra of $\mathfrak{k}^{\prime}$ is not a Cartan subalgebra of $\mathfrak{g}^{\prime}$, then $\mathfrak{g}$ and $\mathfrak{g}^{\prime}$ are not isomorphic. Hence, we assume that $\mathfrak{g}^{\prime}$ is of inner type and define $\mathfrak{h}^{\prime}, \Phi^{\prime}$, and $\Delta^{\prime}$ in the same way. Since $\mathfrak{g}^{c}$ and $\left(\mathfrak{g}^{\prime}\right)^{c}$ are isomorphic we may assume that $\Delta$ and $\Delta^{\prime}$ are ordered so that the corresponding Cartan matrices are the same. Recall that each root space $\mathfrak{g}_{\alpha}$ with $\alpha \in \Phi$ lies either in $\mathfrak{k}^{c}$ or $\mathfrak{p}^{c}$; thus we have $\theta\left(x_{i}\right)=\lambda_{i} x_{i}$ with $\lambda_{i} \in\{ \pm 1\}$ for all $i$. Let $\lambda_{1}^{\prime}, \ldots, \lambda_{l}^{\prime}$ be defined similarly.

Suppose that we are in the situation $\lambda_{i}=\lambda_{i}^{\prime}$ for all $i$, and write $\sigma\left(x_{i}\right)=r_{i} y_{i}$ and $\sigma\left(x_{i}^{\prime}\right)=r_{i}^{\prime} y_{i}^{\prime}$. By Corollary 11, we have $\operatorname{sgn}\left(r_{i}\right)=\operatorname{sgn}\left(r_{i}^{\prime}\right)$ for all $i$, which allows us to define the reals $\mu_{i}=\sqrt{r_{i} / r_{i}^{\prime}}$. Now the isomorphism $\psi: \mathfrak{g}^{c} \rightarrow\left(\mathfrak{g}^{\prime}\right)^{c}$ which maps $\left(h_{i}, x_{i}, y_{i}\right)$ to $\left(h_{i}^{\prime}, \mu_{i} x_{i}^{\prime}, \mu_{i}^{-1} y_{i}^{\prime}\right)$ for all $i$ satisfies $\psi \circ \theta=\theta^{\prime} \circ \psi$ and $\psi \circ \sigma=\sigma^{\prime} \circ \psi$, and we are done.

In the remainder of this section we show how to achieve $\lambda_{i}=\lambda_{i}^{\prime}$ for all $i$ in the case that $\mathfrak{g}$ and $\mathfrak{g}^{\prime}$ are isomorphic. The idea is to use the results of Section 7.1 to 
find a new basis of simple roots such that $\theta$ and its parameters $\lambda_{1}, \ldots, \lambda_{l}$ are in a standard form. As explained below, this means that at most one parameter $\lambda_{k}$ is negative, with certain restrictions on $k$. The possible standard forms are obtained by listing the Kac diagrams of the inner involutions of $\mathfrak{g}^{c}$; to each Kac diagram corresponds exactly one standard form, and $\mathfrak{g}$ and $\mathfrak{g}^{\prime}$ are isomorphic if and only if the standard forms of $\theta$ and $\theta^{\prime}$ coincide.

In the following we explain this in detail for the simple Lie algebra of type $D_{l}$.

Example 13. Let the notation be as above and suppose $\mathfrak{g}^{c}$ is of type $D_{l}$ with $l>4$. We suppose that our basis of simple roots $\Delta=\left\{\alpha_{1}, \ldots, \alpha_{l}\right\}$ corresponds to the labels of the following Dynkin diagram of $D_{l}$ :

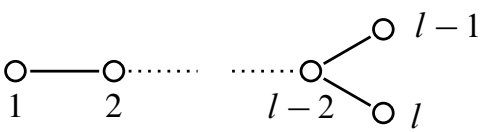

Up to conjugacy, the involutive inner automorphisms of $\mathfrak{g}^{c}$ are $\bar{\chi}_{j}$ with $j=$ $1, \ldots,\lfloor l / 2\rfloor$ or $j=l-1$, where $\bar{\chi}_{j}\left(x_{i}\right)=(-1)^{\delta_{i j}} x_{i}$ for all $i$. If we do not have that $\theta=\bar{\chi}_{j}$ for some $j$, then we proceed as follows. Recall that the parameters of $\theta$ are $\lambda_{1}, \ldots, \lambda_{l}$ where $\theta\left(x_{i}\right)=\lambda_{i} x_{i}$. For $k=1, \ldots, l$ write $w_{k}=s_{\alpha_{k}} \in W$ for the reflection defined by $k$-th simple root $\alpha_{k}$. Let $\tilde{x}_{i}=w_{k}\left(x_{i}\right), \tilde{y}_{i}=w_{k}\left(y_{i}\right)$, and $\tilde{h}_{i}=w_{k}\left(h_{i}\right)$ be the images of the canonical generators under $w_{k}$. As seen in Section 7.1, with respect to this new canonical generating set, $\theta$ has the same parameters as before, except that $\lambda_{j}$ is replaced by $\lambda_{j} \lambda_{k}$ if $\left\langle\alpha_{j}, \alpha_{k}\right\rangle=-1$ (or, equivalently, if $\alpha_{j}$ and $\alpha_{k}$ are connected in the Dynkin diagram). We will now iterate this modification of parameters. We stress that in each iteration step the reflections $w_{1}, \ldots, w_{l}$ are defined with respect to the new basis of simple roots constructed in the previous step; thus, acting with $w_{i}$ and then with $w_{j}$ means we first apply the reflection $s_{\alpha_{i}}$ and then the reflection $s_{w_{i}\left(\alpha_{j}\right)}$. Similarly, in each iteration step we have new parameters $\lambda_{i}$ and a new canonical generating set. By abuse of notation, in each iteration step we always denote these by the same symbols.

We now show that we can apply a sequence of simple reflections to find a new set of canonical generators such that for the parameters of $\theta$ there is a unique $k \in\{1, \ldots,\lfloor l / 2\rfloor, l-1, l\}$ with $\lambda_{k}=-1$; that is, $\theta=\bar{\chi}_{k}$. The details are as follows:

- The first step is to achieve that at most one of $\lambda_{1}, \ldots, \lambda_{l-2}$ has value -1 . If this is not already the case, then there exist $i<k \leq l-2$ with $\lambda_{i}, \lambda_{k}=-1$ and $\lambda_{j}=1$ whenever $i<j<k$ or $k<j \leq l-2$. If we act with $w_{i}, w_{i+1}, \ldots, w_{k-1}$, then we obtain new parameters of $\theta$ with $\lambda_{k-1}=-1$ and $\lambda_{k}=\cdots=\lambda_{l-2}=1$. Now either $k-1$ is the only index in $\{1, \ldots, l-2\}$ with $\lambda_{k-1}=-1$, or we iterate this process. Eventually, at most one value of $\lambda_{1}, \ldots, \lambda_{l-2}$ is -1 . 
- Next, a case distinction with four cases $\lambda_{l-1}, \lambda_{l} \in\{ \pm 1\}$ shows that we can in fact assume that at most one value of $\lambda_{1}, \ldots, \lambda_{l}$ is -1 : For example, suppose $\lambda_{i}=-1$ with $i<l-1$ and $\lambda_{l-1}=-1$ are the only negative parameters. If we act with $w_{l-1}, w_{l-2}, \ldots, w_{i+1}$, then among the new parameters the only negative ones are $\lambda_{i+1}=-1$ and $\lambda_{l}=-1$. By an iteration, the only negative parameters are $\lambda_{l-2}=\lambda_{l}=-1$ (or $\lambda_{l-2}=\lambda_{l-1}=-1$ ), and acting with $w_{l}$ (or $w_{l-1}$ ) yields the assertion.

- If the only negative parameter $\lambda_{k}=-1$ satisfies $k \in\{1, \ldots,\lfloor l / 2\rfloor, l-1, l\}$, then we are done. Thus, suppose we have $\lambda_{k}=-1$ with $\lfloor l / 2\rfloor<k \leq l-2$. Let $t=l-k-1$, and act with $w_{k}, w_{k+1}, \ldots, w_{k+t} ; w_{k-1}, w_{k}, \ldots, w_{k-1+t} ; \ldots$; $w_{1}, w_{2}, \ldots, w_{1+t}$. This gives new parameters with only negative parameter $\lambda_{t+1}=-1$.

If $\lambda_{l}=-1$ is the only negative parameter, then we apply the diagram automorphism which fixes $\alpha_{1}, \ldots, \alpha_{l-1}$ and interchanges $\alpha_{l-1}$ and $\alpha_{l}$; the resulting new basis of simple roots still defines the same Cartan matrix, and now we have $\theta=\bar{\chi}_{l-1}$. Thus, every inner automorphisms $\theta$ of order two can be brought into standard form; that is, there is exactly one negative parameter $\lambda_{k}=-1$, and $k \in\{1, \ldots,\lfloor l / 2\rfloor, l-1\}$.

Our approach for the other simple Lie algebras is the same: We act with the Weyl group (as described in Section 7.1) and certain diagram automorphisms to find a new basis of simple roots such that $\theta$ has standard form; that is, at most one parameter $\lambda_{k}=-1$ is negative, with the following restrictions: $k \leq\lceil l / 2\rceil$ for $A_{l}$, $k=l$ or $k \leq\lfloor l / 2\rfloor$ for $C_{l}, k=1$ for $G_{2}, k \in\{2,3\}$ for $F_{4}, k \in\{1,2\}$ for $E_{6}$, $k \in\{1,2,7\}$ for $E_{7}$, and $k \in\{1,8\}$ for $E_{8}$.

Remark 14. A more uniform approach to the problem of finding the standard form of $\theta$ is by using the classification of finite order inner automorphisms as, for example, given in [Reeder 2010]. In this approach one acts with the affine Weyl group, and finding the Kac diagram of an automorphism is equivalent to finding a point in the fundamental alcove conjugate to a given point. It can be worked out how acting by an element of the affine Weyl group amounts to choosing a different basis of simple roots. For the purposes of this paper, as we are dealing with involutions only, we have chosen the more elementary method outlined above.

7.3. Outer type. Suppose $\theta$ is an outer involutive automorphism of $\mathfrak{g}^{c}$. We apply the following four steps to $\mathfrak{g}$ (and then $\mathfrak{g}^{\prime}$ ).

(1) The first step is to construct a $\theta$-stable Cartan subalgebra of $\mathfrak{g}^{c}$ : For this purpose let $\mathfrak{h}_{0}^{c}$ be a Cartan subalgebra of $\mathfrak{k}^{c}$ and define $\mathfrak{h}^{c}=C_{\mathfrak{g}^{c}}\left(\mathfrak{h}_{0}^{c}\right)$ as its centralizer in $\mathfrak{g}^{c}$. It is shown in [Knapp 2002, Proposition 6.60] that $\mathfrak{h}^{c}$ is a Cartan subalgebra of $\mathfrak{g}^{c}$; clearly, it is fixed by $\theta$. Now $\mathfrak{h}=\mathfrak{h}^{c} \cap \mathfrak{g}$ is a maximally compact Cartan subalgebra of $\mathfrak{g}$ (see [Knapp 2002, Proposition 6.61]), and all 
Cartan subalgebras of $\mathfrak{g}$ constructed this way are conjugate in $\mathfrak{g}$ (see [Knapp 2002, Proposition 6.61]). Thus, if $\mathfrak{g}$ and $\mathfrak{g}^{\prime}$ are isomorphic and $\mathfrak{h}$ and $\mathfrak{h}^{\prime}$ are Cartan subalgebras constructed as above, then there is an isomorphism $\mathfrak{g} \rightarrow \mathfrak{g}^{\prime}$ which maps $\mathfrak{h}$ to $\mathfrak{h}^{\prime}$.

(2) The second step is to construct a basis of simple roots which is stable under $\alpha \mapsto \alpha \circ \theta$ : Let $\Phi$ be the root system with respect to $\mathfrak{h}^{c}$, and recall that, if $\alpha \in \Phi$, then $\beta=\alpha \circ \theta$ is a root with $\theta\left(x_{\alpha}\right) \in \mathfrak{g}_{\beta}$ and $\theta\left(h_{\alpha}\right)=h_{\beta}$. This shows that the $\mathbb{R}$-span $\mathfrak{h}_{\mathbb{R}}$ of all $h_{\alpha}$ with $\alpha \in \Phi$ is invariant under $\theta$. Moreover, $h_{0, \mathbb{R}}=\mathfrak{h}_{\mathbb{R}} \cap \mathfrak{h}_{0}^{c}$ is the 1-eigenspace of $\theta$ in $\mathfrak{h}_{\mathbb{R}}$. Since $\mathfrak{h}_{0, \mathbb{R}}$ spans $\mathfrak{h}_{0}^{c}$ as a $\mathbb{C}$-vector space, the restriction of each $\alpha \in \Phi$ to $\mathfrak{h}_{0, \mathbb{R}}$ is nonzero: if $\alpha\left(\mathfrak{h}_{0, \mathbb{R}}\right)=\{0\}$, then $\mathfrak{g}_{\alpha} \subseteq C_{\mathfrak{g}^{c}}\left(\mathfrak{h}_{0}^{c}\right)=\mathfrak{h}^{c}$ yields a contradiction. This shows that there is $h_{0} \in \mathfrak{h}_{0, \mathbb{R}}$ with $\alpha\left(h_{0}\right) \neq 0$ for all $\alpha \in \Phi$ : such an $h_{0}$ can be chosen as any element outside a finite number of hyperplanes in $\mathfrak{h}_{0, \mathbb{R}}$, namely, the kernels of $\alpha$ in $h_{0, \mathbb{R}}$. We use $h_{0}$ to define $\alpha>0$ if and only if $\alpha\left(h_{0}\right)>0$; note that elements in $\mathfrak{h}_{\mathbb{R}}$ only have real eigenvalues. It is easy to check that this defines a root ordering, and, if $\alpha>0$, then also $\alpha \circ \theta>0$. Therefore the corresponding set of simple roots $\Delta=\left\{\alpha_{1}, \ldots, \alpha_{l}\right\}$ is $\theta$-stable. Let $\pi$ be the permutation of $\{1, \ldots, l\}$ defined by $\alpha_{i} \circ \theta=\alpha_{\pi(i)}$, and denote by $\left\{h_{i}, x_{i}, y_{i} \mid i\right\}$ a canonical generating set corresponding to $\Delta$.

(3) The third step is to adjust the coefficients of $\theta$ : If $\pi(i)=i$, then set $\tilde{h}_{i}=h_{i}$, $\tilde{x}_{i}=x_{i}$ and $\tilde{y}_{i}=y_{i}$. Otherwise, for all $(i, \pi(i))$ with $\pi(i)>i$ set $\tilde{h}_{i}=h_{i}$, $\tilde{x}_{i}=x_{i}, \tilde{y}_{i}=y_{i}$, and $\tilde{h}_{\pi(i)}=\theta\left(h_{i}\right), \tilde{x}_{\pi(i)}=\theta\left(x_{i}\right), \tilde{y}_{\pi(i)}=\theta\left(y_{i}\right)$. Вy replacing $\left\{h_{i}, x_{i}, y_{i} \mid i\right\}$ with the canonical generating set $\left\{\tilde{h}_{i}, \tilde{x}_{i}, \tilde{y}_{i} \mid i\right\}$ (see Proposition 3), we may assume that $\theta\left(x_{i}\right)=\lambda_{i} x_{\pi(i)}$ with $\lambda_{i}=1$ if $\pi(i) \neq i$.

(4) Finally, we decompose $\theta$ : Let $\varphi$ be the diagram automorphism defined by $\pi$ with respect to the new canonical generating set defined in (3) (see Section 2.2); that is, $\varphi\left(x_{i}\right)=x_{\pi(i)}, \varphi\left(y_{i}\right)=y_{\pi(i)}$, and $\varphi\left(h_{i}\right)=h_{\pi(i)}$ for all $i$. By construction,

$$
\chi=\varphi \circ \theta=\theta \circ \varphi
$$

is an involutive inner automorphism of $\mathfrak{g}^{c}$ with $\chi\left(x_{i}\right)=x_{i}$ if $\pi(i) \neq i$, and $\chi\left(x_{i}\right)=\lambda_{i} x_{i}$ if $\pi(i)=i$ and $\theta\left(x_{i}\right)=\lambda_{i} x_{i}$; clearly, $\lambda_{i}= \pm 1$. The analogous statement holds for $y_{i}$.

We use the same procedure to construct a $\theta^{\prime}$-stable set of positive roots $\Delta^{\prime}$, and automorphisms $\varphi^{\prime}$ and $\chi^{\prime}$ of $\left(\mathfrak{g}^{\prime}\right)^{c}$. We also assume that the bases $\Delta$ and $\Delta^{\prime}$ are ordered such that the corresponding Cartan matrices are the same, and $\pi=\pi^{\prime}$ as permutations of $\{1, \ldots, l\}$. If the latter is not possible, then $\mathfrak{g}^{c}$ and $\left(\mathfrak{g}^{\prime}\right)^{c}$ are not isomorphic. Let $\left\{h_{i}, x_{i}, y_{i} \mid i\right\}$ and $\left\{h_{i}^{\prime}, x_{i}^{\prime}, y_{i}^{\prime} \mid i\right\}$ be the associated sets of canonical 
generators as constructed in Step (3) above, and let $\psi: \mathfrak{g}^{c} \rightarrow\left(\mathfrak{g}^{\prime}\right)^{c}$ be the associated isomorphism. We now try to modify $\psi$ so that it is compatible with $\theta, \theta^{\prime}$ and $\sigma, \sigma^{\prime}$.

7.3.1. Make $\psi$ compatible with $\theta$ and $\theta^{\prime}$. Recall that $\theta=\chi \circ \varphi, \theta^{\prime}=\chi^{\prime} \circ \varphi^{\prime}$, and $\psi \circ \varphi=\varphi^{\prime} \circ \psi$. If $\pi(i) \neq i$, then

$$
\theta^{\prime} \circ \psi\left(x_{i}\right)=\theta^{\prime}\left(x_{i}^{\prime}\right)=x_{\pi^{\prime}(i)}^{\prime}=x_{\pi(i)}^{\prime}=\psi\left(x_{\pi(i)}\right)=\psi \circ \theta\left(x_{i}\right)
$$

similarly, $\theta^{\prime} \circ \psi$ and $\psi \circ \theta$ coincide on the whole subspace of $\mathfrak{g}^{c}$ spanned by all $x_{i}, y_{i}, h_{i}$ with $\pi(i) \neq i$. If $\pi(i)=i$ with $\theta\left(x_{i}\right)=\lambda_{i} x_{i}$ and $\theta^{\prime}\left(x_{i}^{\prime}\right)=\lambda_{i}^{\prime} x_{i}^{\prime}$, then $\theta^{\prime} \circ \psi\left(x_{i}\right)=\psi \circ \theta\left(x_{i}\right)$ if and only if $\lambda_{i}=\lambda_{i}^{\prime}$.

- Type $A_{l}$ : If $l=2 m$ is even, then $\pi$ acts fixed-point freely; thus $\psi$ as constructed above already satisfies $\theta^{\prime} \circ \psi=\psi \circ \theta$. If $l=2 m+1$, then $\pi$ has exactly one fixed point, say $i=1$, and either $\lambda_{1}=1$ or $\lambda_{1}=-1$. On the other hand, up to conjugacy, $A_{l}$ has two outer automorphisms, so each choice for $\lambda_{1}$ corresponds to a different conjugacy class of automorphisms. Thus, if $\mathfrak{g}^{c}$ and $\left(\mathfrak{g}^{\prime}\right)^{c}$ are isomorphic, then $\lambda_{1}=\lambda_{1}^{\prime}$, and $\psi$ is an isomorphism with $\theta^{\prime} \circ \psi=\psi \circ \theta$.

- Type $E_{6}$ : Here $\pi$ has two fixed points, say $i=2,4$; thus there are four possible combinations of signs for $\lambda_{2}$ and $\lambda_{4}$. However, up to conjugacy, $E_{6}$ has two outer automorphisms. Suppose our root basis $\Delta=\left\{\alpha_{1}, \ldots, \alpha_{6}\right\}$ corresponds to the labels of the following Dynkin diagram of $E_{6}$ :

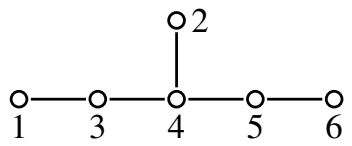

Up to conjugacy, $E_{6}$ has two outer automorphisms $\varphi \circ \bar{\chi}$, where $\varphi$ is the diagram automorphism acting via the permutation $\pi=(1,6)(3,5)$, and $\bar{\chi}$ is an inner automorphisms which satisfies $\bar{\chi}\left(x_{4}\right)= \pm x_{4}$ and $\bar{\chi}\left(x_{i}\right)=x_{i}$ if $i \neq 4$. As outlined in Section 7.1 we now act with $w_{2}=s_{\alpha_{2}}$ and $w_{4}=s_{\alpha_{4}}$ in order to find a new canonical generating set (with respect to a new basis of simple roots), relative to which we have $\lambda_{2}=\lambda_{4}=1$, or $\lambda_{2}=1$ and $\lambda_{4}=-1$. It is straightforward to see that this can always be done. For example, if $\lambda_{2}=-1$ and $\lambda_{4}=1$, then we first act with $w_{2}$ to get $\lambda_{2}=\lambda_{4}=-1$ and subsequently with $w_{4}$ to get $\lambda_{2}=1$ and $\lambda_{4}=-1$. Finally we use the same trick as in the beginning of Section 7.3 to obtain $\lambda_{i}=1$ for all $i \neq 2,4$ (that is, we set $x_{5}=\theta\left(x_{3}\right)$, etc.). The conclusion is that we can arrange that $\lambda_{i}=\lambda_{i}^{\prime}$ for every $i$; hence $\psi$ is an isomorphism with $\theta^{\prime} \circ \psi=\psi \circ \theta$.

- Type $D_{l}$ : We proceed as for $E_{6}$ and suppose that our basis of simple roots $\Delta$ corresponds to the Dynkin diagram of $D_{l}$ as shown on page 370. Up to conjugacy, the involutive outer automorphisms of $D_{l}$ are $\varphi \circ \bar{\chi}$, where $\varphi$ is the diagram automorphism defined by $\pi=(l-1, l)$, and $\bar{\chi}$ is an inner 
automorphism with $\bar{\chi}\left(x_{i}\right)=\bar{\lambda}_{i} x_{i}$, where either $\bar{\lambda}_{i}=1$ for all $i$, or there exists a unique negative $\bar{\lambda}_{k}$ and $k \in\{1, \ldots,\lceil l / 2\rceil-1\}$. As in Example 13, we act with reflections $s_{\alpha_{j}} \in W, j \in\{1, \ldots, l-2\}$, to find a canonical generating set relative to which there is a unique negative parameter $\lambda_{k}$, and $k \in\{1, \ldots, l-2\}$. If $k \leq\lceil l / 2\rceil-1$, then we are done; otherwise we proceed as follows. Set $\beta_{i}=\alpha_{l-i-1}$ for $i=1, \ldots, l-2$, and $\beta_{l-1}=-\alpha_{1}-\cdots-\alpha_{l-2}-\alpha_{l-1}$, and $\beta_{l}=-\alpha_{1}-\cdots-\alpha_{l-2}-\alpha_{l}$. Then $\bar{\Delta}=\left\{\beta_{1}, \ldots, \beta_{l}\right\}$ is also a basis of simple roots with the same Dynkin diagram. Now we take a canonical generating set with respect to $\bar{\Delta}$. With respect to this new canonical generating set, $\chi$ has a unique negative parameter $\lambda_{k}=-1$, and $k \in\{1, \ldots,\lceil l / 2\rceil-1\}$.

Using these constructions, we can arrange that $\lambda_{i}=\lambda_{i}^{\prime}$ for all $i$; thus the corresponding isomorphism $\psi$ (defined on the newly constructed canonical generating sets) is compatible with $\theta$ and $\theta^{\prime}$.

7.3.2. Make $\psi$ compatible with $\sigma$ and $\sigma^{\prime}$. Using the construction in the previous paragraphs, we have established that either $\mathfrak{g}$ and $\mathfrak{g}^{\prime}$ are not isomorphic, or we have an isomorphism $\psi: \mathfrak{g}^{c} \rightarrow\left(\mathfrak{g}^{\prime}\right)^{c}$ compatible with $\theta$ and $\theta^{\prime}$. We assume the latter holds, and we now adjust $\psi$ so it is also compatible with the complex conjugations $\sigma$ and $\sigma^{\prime}$; this yields the desired isomorphism between $\mathfrak{g}$ and $\mathfrak{g}^{\prime}$.

By our previous construction, if $i \neq \pi(i)$, then $\theta\left(x_{i}\right)=x_{\pi(i)}$, and $\theta\left(x_{i}\right)=\lambda_{i} x_{i}$ otherwise. Lemma 7 shows that $\sigma\left(x_{i}\right)=r_{i} y_{\pi(i)}$ for some $r_{i} \in \mathbb{R}$ with $r_{i}=r_{\pi(i)}$. If $i \neq \pi(i)$, then $r_{i}<0$, and, if $i=\pi(i)$, then $r_{i}$ and $-\lambda_{i}$ have the same sign; see Corollary 11. Now define $\mu_{i}=\sqrt{1 /\left|r_{i}\right|}$ for $i=1, \ldots, l$. If we replace $x_{i}, y_{i}, x_{\pi(i)}, y_{\pi(i)}$ by $\tilde{x}_{i}=\mu_{i} x_{i}, \tilde{y}_{i}=\mu_{i}^{-1} y_{i}, \tilde{x}_{\pi(i)}=\mu_{i} x_{\pi(i)}, \tilde{y}_{\pi(i)}=\mu_{i}^{-1} y_{\pi(i)}$, then we get a new set of canonical generators where $\theta$ acts in the same way and $\sigma\left(\tilde{x}_{i}\right)= \pm \tilde{y}_{\pi(i)}$ for all $i$. In a similar way, we obtain a new set of canonical generators $\left\{\tilde{x}_{i}^{\prime}, \tilde{y}_{i}^{\prime}, h_{i}^{\prime} \mid i\right\}$ of $\left(\mathfrak{g}^{\prime}\right)^{c}$; recall that $\lambda_{i}=\lambda_{i}^{\prime}$ for all $i$. The associated isomorphism $\mathfrak{g}^{c} \rightarrow\left(\mathfrak{g}^{\prime}\right)^{c}$ now is compatible with $\theta, \sigma$, and $\theta^{\prime}, \sigma^{\prime}$, and restricts to an isomorphism $\mathfrak{g} \rightarrow \mathfrak{g}^{\prime}$ preserving the Cartan decompositions.

Remark 15. In the algorithms described in this section we compute root systems of $\mathfrak{g}^{c}$ and $\left(\mathfrak{g}^{\prime}\right)^{c}$ with respect to Cartan subalgebras $\mathfrak{h}^{c}$ and $\left(\mathfrak{h}^{\prime}\right)^{c}$. In order for that to work well we need Cartan subalgebras that split over $\mathbb{Q}^{\sqrt{ }}(l)$ (or an extension thereof of small degree). However, the problem of finding such Cartan subalgebras is very difficult; see [Ivanyos et al. 2012]. Therefore, in our algorithms we assume that we have a Cartan subalgebra with a small splitting field.

7.4. Nilpotent orbits under isomorphisms. Suppose $\mathfrak{g}=\mathfrak{k} \oplus \mathfrak{p}$ and $\mathfrak{g}^{\prime}=\mathfrak{k}^{\prime} \oplus \mathfrak{p}^{\prime}$ are semisimple real Lie algebras and $\psi: \mathfrak{g} \rightarrow \mathfrak{g}^{\prime}$ is an isomorphism such that $\psi(\mathfrak{k})=\mathfrak{k}^{\prime}$ and $\psi(\mathfrak{p})=\mathfrak{p}^{\prime}$. As described in the previous sections, we can extend this to an isomorphism $\psi: \mathfrak{g}^{c} \rightarrow(\mathfrak{g})^{c}$ compatible with the corresponding Cartan involutions $\theta, \theta^{\prime}$ 
and complex conjugations $\sigma, \sigma^{\prime}$. Let $G$ be the connected Lie subgroup of the adjoint group $G^{c}$ of $\mathfrak{g}^{c}$, having Lie algebra $\mathfrak{g}$. Similarly, let $G^{\prime}$ be defined for $\mathfrak{g}^{\prime}$.

Lemma 16. The isomorphism $\psi: \mathfrak{g} \rightarrow \mathfrak{g}^{\prime}$ maps nilpotent orbits to nilpotent orbits.

Proof. Clearly, $e \in \mathfrak{g}$ is nilpotent if and only if $\psi(e) \in \mathfrak{g}^{\prime}$ is nilpotent. We show that, if two nilpotent $e, f \in \mathfrak{g}$ are conjugate under $G$, then $e^{\prime}=\psi(e)$ and $f^{\prime}=$ $\psi(f)$ are conjugate under $G^{\prime}$; then the same argument with $\psi$ replaced by $\psi^{-1}$ proves the assertion. As shown in [Helgason 1978, pp. 126-127], the adjoint group $G$ is generated by all exp ad $x$ with $x \in \mathfrak{g}$, and the isomorphism $\psi$ lifts to an isomorphism $\tilde{\psi}: G \rightarrow G^{\prime}, \beta \mapsto \psi \circ \beta \circ \psi^{-1}$. Thus, if $\beta(e)=f$ for some $\beta \in G$, then $\tilde{\psi}(\beta)(\psi(e))=\psi(f)$, and $\psi(e)$ and $\psi(f)$ are $G^{\prime}$-conjugate in $\mathfrak{g}^{\prime}$.

\section{Appendix: Comment on the implementation}

For computing with semisimple Lie algebras we use the package SLA [de Graaf 2012] for the computer algebra system GAP [GAP 2012]. This package provides the functionality, for example, to compute Chevalley bases, canonical generators, and involutive automorphisms. In Section 6.3 we use the Gröbner bases functionality of the computer algebra system SINGULAR [Decker et al. 2011] via the linkage package Singular [Costantini and de Graaf 2006].

A.1. The field $\mathbb{Q} \sqrt{ }$. We now comment on the field $\mathbb{Q} \sqrt{ }=\mathbb{Q}(\{\sqrt{p} \mid p$ a prime $\})$. GAP already allows us to work with subfields of cyclotomic fields $\mathbb{Q}\left(\zeta_{n}\right)$, where $\zeta_{n}$ is a complex primitive $n$-th root of unity. However, if $x=\sum_{i=1}^{m} \sqrt{p_{i}}$ for primes $p_{1}, \ldots, p_{m}$, then the smallest $n$ with $x \in \mathbb{Q}\left(\zeta_{n}\right)$ is $n=\operatorname{lcm}\left(e_{1}, \ldots, e_{m}\right)$, where $e_{i}=p_{i}$ if $p_{i} \equiv 1 \bmod 4$, and $e_{i}=4 p_{i}$ otherwise; see Lemma 17. Thus, already for small $m$ this requires to work in large cyclotomic fields. Alternatively, one could work in an algebraic extension defined by an irreducible polynomial over $\mathbb{Q}$. The disadvantage here is that we do not know in the beginning which irrationals turn up, so we would have to extend and therefore change the underlying field several times. To avoid all this, we have implemented our own realization of $\mathbb{Q} \sqrt{ }(l)$. Every element of $\mathbb{Q} \sqrt{ }(l)$ can be written uniquely as $u=\sum_{i=1}^{n} r_{i} \sqrt{z_{i}}$ where $z_{i}>0$ are pairwise distinct squarefree integers and $r_{i} \in \mathbb{Q}(l)$. Internally, we represent $u$ as a list with entries $\left(r_{i}, z_{i}\right)$, which allows efficient addition and multiplication in $\mathbb{Q} \sqrt{ }(l)$. A computational bottleneck is the construction of the multiplicative inverse of such a $u \neq 0$ : We compute powers $\left\{1, u, u^{2}, \ldots, u^{m}\right\}$ until $u^{m}$ can be expressed as a $\mathbb{Q}$-linear combination of $\left\{1, u, \ldots, u^{m-1}\right\}$, say $u^{m}=\sum_{i=0}^{m-1} q_{i} u^{i}$. The minimal polynomial of $u$ over $\mathbb{Q}$ is $f(x)=x^{m}-\sum_{i=0}^{m-1} q_{i} x^{i}=x g(x)+q_{0}$; therefore $u^{-1}=-g(u) / q_{0}$. Although all this can done with linear algebra, $m$ can become rather large; see Lemma 19. 
Often we had to deal with the following problem: Suppose $v \in \mathbb{Q} \sqrt{ }(l)$ is given as an element of $\mathbb{Q}\left(\zeta_{n}\right)$ for some $n$; write it as an element of $\mathbb{Q} \sqrt{ }(l)$, that is, $v=\sum_{i=1}^{m} r_{i} \sqrt{k_{i}}$ for pairwise distinct positive squarefree integers $k_{i}$ and Gaussian rationals $r_{i}$. Clearly, it is sufficient to consider $v$ real. The first step is to determine the set $\mathscr{S}$ of all positive squarefree $k$ with $\sqrt{k} \in \mathbb{Q}\left(\zeta_{n}\right)$; we do this in Corollary 18 . The second step is to make the ansatz $v=\sum_{k \in \mathscr{S}} r_{k} \sqrt{k}$ in $\mathbb{Q}\left(\zeta_{n}\right)$ with indeterminates $r_{k} \in \mathbb{Q}$. Linear algebra can be used to find a solution of this equation; we prove in Lemma 19 that such a solution always exists. We now provide the theoretical background of this approach; our starting point is the following lemma; see [Shirali and Yogananda 2004, p. 56, Proposition 3], and its corollary.

Lemma 17. If $p$ is an odd prime, then $\sqrt{(-1)^{(p-1) / 2} p} \in \mathbb{Q}\left(\zeta_{p}\right)$.

Corollary 18. Let $k$ and $n$ be positive integers. Suppose $k$ is squarefree and let $e$ be the number of primes $p \equiv 3 \bmod 4$ dividing $k$.

(a) If $\sqrt{2} \in \mathbb{Q}\left(\zeta_{n}\right)$, then $8 \mid n$. If $\sqrt{k} \in \mathbb{Q}\left(\zeta_{n}\right)$, then $k \mid n$.

(b) If $n$ is odd, then $\mathbb{Q}\left(\zeta_{n}\right)=\mathbb{Q}\left(\zeta_{2 n}\right)$, and $\sqrt{k} \in \mathbb{Q}\left(\zeta_{n}\right)$ if and only if e is even and $k \mid n$.

(c) If $4 \mid n$ and $8 \nmid n$, then $\sqrt{k} \in \mathbb{Q}\left(\zeta_{n}\right)$ if and only if $k$ is odd and $k \mid n$.

(d) If $8 \mid n$, then $\sqrt{k} \in Q\left(\zeta_{n}\right)$ if and only if $k \mid n$.

(e) Let $n$ be minimal with $\sqrt{k} \in \mathbb{Q}\left(\zeta_{n}\right)$. If $k$ is odd and e is even, then $n=k$, and $n=4 k$ otherwise.

Lemma 19. (a) Let $n, k_{1}, \ldots, k_{m}$ be pairwise distinct positive squarefree integers and suppose there exists a prime $p \mid n$ with $p \nmid k_{i}$ for all $i$. Then $\sqrt{n} \notin$ $\mathbb{Q}\left(\sqrt{k_{1}}, \ldots, \sqrt{k_{m}}\right)$.

(b) Let $v=\sum_{i=1}^{m} r_{i} \sqrt{k_{i}} \in \mathbb{Q} \sqrt{ }$ for rational $r_{i} \neq 0$ and pairwise distinct positive squarefree integers $k_{i}$. Then $v$ is a primitive element of $\mathbb{Q}\left(\sqrt{k_{1}}, \ldots, \sqrt{k_{m}}\right)$.

Proof. (a) We use induction on $m$. The assertion clearly holds if $m=1$; thus let $m>1$ and write $K^{\prime}=\mathbb{Q}\left(\sqrt{k_{1}}, \ldots, \sqrt{k_{m-1}}\right)$ and $K=K^{\prime}(b)$ with $b=\sqrt{k_{m}}$. Suppose that $\sqrt{n} \in K$. Since $\sqrt{n} \notin K^{\prime}$ by the induction hypothesis, $b \notin K^{\prime}$ and, therefore, $\sqrt{n}=r+b s$ for unique $r, s \in K^{\prime}$. Note that $s, r \neq 0$ since otherwise $\sqrt{n}$ or $\sqrt{n k_{m}} / k_{m}$ would lie in $K^{\prime}$, a contradiction. Now squaring yields $b=$ $\left(n-r^{2}-s^{2} k_{m}\right) /(2 r s) \in K^{\prime}$, the final contradiction.

(b) Suppose $K=\mathbb{Q}\left(\sqrt{k_{1}}, \ldots, \sqrt{k_{m}}\right)=\mathbb{Q}\left(\sqrt{k_{1}}, \ldots, \sqrt{k_{s}}\right)$ has degree $d=2^{s}$ over $\mathbb{Q}$ with $s \leq m$. Since $K$ is the splitting field of the separable polynomial $\left(x^{2}-k_{1}\right) \cdots\left(x^{2}-k_{s}\right)$, the extension is Galois and therefore $\varphi=\operatorname{Gal}(K / \mathbb{Q})$ has order $d$. Clearly, every map defined by $\sqrt{k_{i}} \mapsto \pm \sqrt{k_{i}}$ for $i=1, \ldots, s$ gives rise to a Galois automorphism, and an order argument shows that $\mathscr{G}$ consists exactly of these automorphisms. We now show that $1, \sqrt{k_{1}}, \ldots, \sqrt{k_{s}}$ are linearly independent 
over $\mathbb{Q}$. Clearly, this is true for $s=1$, so let $s \geq 2$. For a contradiction, assume (†) $\sum_{i=1}^{s} r_{i} \sqrt{k_{i}}+r_{m+1}=0$ for rationals $r_{i}$. Let $p$ be a prime dividing $k_{1} \cdots k_{s}$. Now $(\dagger)$ implies that $\sqrt{p}$ lies in the field generated by $\sqrt{k_{1}^{\prime}}, \ldots, \sqrt{k_{s}^{\prime}}$ with $k_{i}^{\prime}=$ $k_{i} / \operatorname{gcd}\left(k_{i}, p\right)$, contradicting part (a). Let $f$ be the minimal polynomial of $v$ over $\mathbb{Q}$. Clearly, $\gamma(v)$ is a root of $f$ for every $\gamma \in \mathscr{G}$. Since $\sqrt{k_{1}}, \ldots, \sqrt{k_{s}}$ are $\mathbb{Q}$-linearly independent, it follows that $\gamma(v) \neq \gamma^{\prime}(v)$ for all $\gamma \neq \gamma^{\prime}$ in $\mathscr{G}$. This shows that $f$ has at least $d$ different zeros, which implies that $f$ has in fact degree $d$ and $v$ is primitive.

\section{References}

[Bourbaki 1975] N. Bourbaki, Éléments de mathématique: Fasc. XXXVIII: Groupes et algèbres de Lie. Chapitre VII: Sous-algèbres de Cartan, éléments réguliers. Chapitre VIII: Algèbres de Lie semi-simples déployées, Actualités Scientifiques et Industrielles 1364, Hermann, Paris, 1975. MR 56 \#12077 Zbl 0329.17002

[Collingwood and McGovern 1993] D. H. Collingwood and W. M. McGovern, Nilpotent orbits in semisimple Lie algebras, Van Nostrand Reinhold, New York, 1993. MR 94j:17001 Zbl 0972.17008

[CoReLG 2012] H. Dietrich, P. Faccin, and W. A. de Graaf, "CoReLG - computing with real Lie groups", a GAP 4 package, 2012, Available at http://www.science.unitn.it/ corelg.

[Costantini and de Graaf 2006] M. Costantini and W. A. de Graaf, "Singular: a GAP 4 package", The GAP Group, 2006, Available at http://www.gap-system.org/Packages/singular.html.

[Cox et al. 1992] D. Cox, J. Little, and D. O'Shea, Ideals, varieties, and algorithms: an introduction to computational algebraic geometry and commutative algebra, Springer, New York, 1992. MR 93j:13031 Zbl 0756.13017

[Decker et al. 2011] W. Decker, G.-M. Greuel, G. Pfister, and M. Schönemann, "Singular: a computer algebra system for polynomial computations", 2011, Available at http://www.singular.uni-kl.de. Version 3-1-3.

[Dietrich et al. 2013] H. Dietrich, P. Faccin, and W. A. de Graaf, "Computing with real Lie algebras: Real forms, Cartan decompositions, and Cartan subalgebras", J. Symbolic Comput. 56 (2013), 27-45. MR 3061707

[Đoković 1987] D. Ž. Đoković, "Proof of a conjecture of Kostant”, Trans. Amer. Math. Soc. 302:2 (1987), 577-585. MR 88j:17007 Zbl 0631.17004

[Đoković 1988] D. Ž. Đoković, "Classification of nilpotent elements in simple exceptional real Lie algebras of inner type and description of their centralizers", J. Algebra 112:2 (1988), 503-524. MR 89b:17010 Zbl 0639.17005

[Đoković 1998] D. Ž. Đoković, "Explicit Cayley triples in real forms of $G_{2}, F_{4}$ and $E_{6}$ ", Pacific J. Math. 184:2 (1998), 231-255. MR 99e:17005 Zbl 1040.17004

[Đoković 1999] D. Ž. Đoković, "Explicit Cayley triples in real forms of $E_{7}$ ", Pacific J. Math. 191:1 (1999), 1-23. MR 2001c:22012 Zbl 1040.17006

[Đoković 2000] D. Ž. Đoković, "Explicit Cayley triples in real forms of $E_{8}$ ", Pacific J. Math. 194:1 (2000), 57-82. MR 2001c:22013 Zbl 1013.22003

[Galina 2009] E. Galina, "Weighted Vogan diagrams associated to real nilpotent orbits", pp. 239-253 in New developments in Lie theory and geometry (Córdoba, 2007), edited by C. S. Gordon et al., Contemp. Math. 491, Amer. Math. Soc., Providence, RI, 2009. MR 2010m:17007 Zbl 1180.22017 
[GAP 2012] The GAP Group, "GAP: groups, algorithms, and programming”, 2012, Available at http://www.gap-system.org. Version 4.5.5.

[de Graaf 2000] W. A. de Graaf, Lie algebras: theory and algorithms, North-Holland Mathematical Library 56, North-Holland, Amsterdam, 2000. MR 2001j:17011 Zbl 1122.17300

[de Graaf 2011] W. A. de Graaf, "Computing representatives of nilpotent orbits of $\theta$-groups", $J$. Symbolic Comput. 46:4 (2011), 438-458. MR 2012f:17008 Zbl 1222.17002

[de Graaf 2012] W. A. de Graaf, "SLA: computing with simple Lie algebras, a GAP 4 package", 2012, Available at http://www.science.unitn.it/ degraaf/sla.html.

[Helgason 1978] S. Helgason, Differential geometry, Lie groups, and symmetric spaces, Pure and Applied Mathematics 80, Academic Press, New York, 1978. MR 80k:53081 Zbl 0451.53038

[Humphreys 1978] J. E. Humphreys, Introduction to Lie algebras and representation theory, 2nd ed., Graduate Texts in Mathematics 9, Springer, New York, 1978. MR 81b:17007 Zbl 0447.17001

[Ivanyos et al. 2012] G. Ivanyos, L. Rónyai, and J. Schicho, "Splitting full matrix algebras over algebraic number fields”, J. Algebra 354 (2012), 211-223. MR 2879232 Zbl 06078371

[Jacobson 1962] N. Jacobson, Lie algebras, Interscience Tracts in Pure and Applied Mathematics 10, Wiley Interscience, New York, 1962. MR 26 \#1345 Zbl 0121.27504

[Kac 1990] V. G. Kac, Infinite-dimensional Lie algebras, 3rd ed., Cambridge University Press, Cambridge, 1990. MR 92k:17038 Zbl 0716.17022

[Knapp 2002] A. W. Knapp, Lie groups beyond an introduction, 2nd ed., Progress in Mathematics 140, Birkhäuser, Boston, 2002. MR 2003c:22001 Zbl 1075.22501

[Kostant and Rallis 1971] B. Kostant and S. Rallis, "Orbits and representations associated with symmetric spaces”, Amer. J. Math. 93 (1971), 753-809. MR 47 \#399 Zbl 0224.22013

[Littelmann 1996] P. Littelmann, “An effective method to classify nilpotent orbits", pp. 255-269 in Algorithms in algebraic geometry and applications (Santander, 1994), edited by L. González-Vega and T. Recio, Progress in Mathematics 143, Birkhäuser, Basel, 1996. MR 98a:20041 Zbl 0866.20037

[Noël 1998] A. G. Noël, "Nilpotent orbits and theta-stable parabolic subalgebras", Represent. Theory 2 (1998), 1-32. MR 99g:17023 Zbl 0891.17006

[Noël 2001a] A. G. Noël, "Classification of admissible nilpotent orbits in simple exceptional real Lie algebras of inner type", Represent. Theory 5 (2001), 455-493. MR 2002i:17015a Zbl 1005.17006

[Noël 2001b] A. G. Noël, "Classification of admissible nilpotent orbits in simple real Lie algebras $E_{6(6)}$ and $E_{6(-26)}$ ", Represent. Theory 5 (2001), 494-502. MR 2002i:17015b Zbl 1005.17005

[Onishchik 2004] A. L. Onishchik, Lectures on real semisimple Lie algebras and their representations, European Mathematical Society, Zürich, 2004. MR 2005b:17014 Zbl 1080.17001

[Reeder 2010] M. Reeder, “Torsion automorphisms of simple Lie algebras”, Enseign. Math. (2) 56:1-2 (2010), 3-47. MR 2012b:17040 Zbl 1223.17020

[Rothschild 1972] L. P. Rothschild, "Orbits in a real reductive Lie algebra”, Trans. Amer. Math. Soc. 168 (1972), 403-421. MR 50 \#2271 Zbl 0222.17009

[Sekiguchi 1987] J. Sekiguchi, "Remarks on real nilpotent orbits of a symmetric pair", J. Math. Soc. Japan 39:1 (1987), 127-138. MR 88g:53053 Zbl 0627.22008

[Shirali and Yogananda 2004] S. Shirali and C. S. Yogananda, Number theory, Universities Press, Hyderabad, 2004.

[Vinberg 1979] È. B. Vinberg, "Classification of homogeneous nilpotent elements of a semisimple graded Lie algebra”, Trudy Sem. Vektor. Tenzor. Anal. 19 (1979), 155-177. In Russian; translated in Selecta Mathematica Sovietica 6:1 (1987), 15-35. MR 80k:17006 Zbl 0431.17006 
[Vinberg et al. 1990] ̇. B. Vinberg, V. V. Gorbatsevich, and A. L. Onishchik, Строение групп и алгебр ли, Encyclopaedia of Mathematical Sciences 41, Akad. Nauk SSSR, Vsesoyuz. Inst. Nauchn. i Tekhn. Inform., Moscow, 1990. In Russian; translated as Lie groups and Lie algebras, III: Structure of Lie groups and Lie algebras, by V. V. Minakhin, Springer, Berlin, 1994. MR 91b:22001 Zbl 0733.22003

Received July 27, 2012. Revised September 17, 2012.

HEIKO DIETRICH

SCHOOL OF MATHEMATICAL SCIENCES

MONASH UNIVERSITY

Clayton, VIC 3800

Australia

heiko.dietrich@monash.edu

WILLEM A. DE GRAAF

DEPARTMENT OF MATHEMATICS

UNIVERSITY OF TRENTO

I-38050 Povo

ITALY

degraaf@science.unitn.it 


\title{
PACIFIC JOURNAL OF MATHEMATICS
}

\author{
msp.org/pjm
}

Founded in 1951 by E. F. Beckenbach (1906-1982) and F. Wolf (1904-1989)

\section{EDITORS}

V. S. Varadarajan (Managing Editor)

Department of Mathematics

University of California

Los Angeles, CA 90095-1555

pacific@math.ucla.edu

Paul Balmer

Department of Mathematics

University of California

Los Angeles, CA 90095-1555

balmer@math.ucla.edu

Daryl Cooper

Department of Mathematics

University of California

Santa Barbara, CA 93106-3080 cooper@math.ucsb.edu

Jiang-Hua $\mathrm{Lu}$

Department of Mathematics

Pokfulam Rd., Hong Kong jhlu@maths.hku.hk
The University of Hong Kong

Don Blasius

Department of Mathematics University of California

Los Angeles, CA 90095-1555

blasius@math.ucla.edu

Robert Finn

Department of Mathematics Stanford University

Stanford, CA 94305-2125

finn@math.stanford.edu

Sorin Popa

Department of Mathematics

University of California

Los Angeles, CA 90095-1555 popa@math.ucla.edu

Paul Yang

Department of Mathematics Princeton University

Princeton NJ 08544-1000

yang@math.princeton.edu

\section{PRODUCTION}

Silvio Levy, Scientific Editor, production@msp.org

\section{SUPPORTING INSTITUTIONS}

ACADEMIA SINICA, TAIPEI

CALIFORNIA INST. OF TECHNOLOGY

INST. DE MATEMÁTICA PURA E APLICADA

KEIO UNIVERSITY

MATH. SCIENCES RESEARCH INSTITUTE

NEW MEXICO STATE UNIV.

OREGON STATE UNIV.

\author{
STANFORD UNIVERSITY \\ UNIV. OF BRITISH COLUMBIA \\ UNIV. OF CALIFORNIA, BERKELEY \\ UNIV. OF CALIFORNIA, DAVIS \\ UNIV. OF CALIFORNIA, LOS ANGELES \\ UNIV. OF CALIFORNIA, RIVERSIDE \\ UNIV. OF CALIFORNIA, SAN DIEGO \\ UNIV. OF CALIF., SANTA BARBARA
}

\author{
Vyjayanthi Chari \\ Department of Mathematics \\ University of California \\ Riverside, CA 92521-0135 \\ chari@math.ucr.edu \\ Kefeng Liu \\ Department of Mathematics \\ University of California \\ Los Angeles, CA 90095-1555 \\ liu@math.ucla.edu \\ Jie Qing \\ Department of Mathematics \\ University of California \\ Santa Cruz, CA 95064 \\ qing@cats.ucsc.edu
}

These supporting institutions contribute to the cost of publication of this Journal, but they are not owners or publishers and have no responsibility for its contents or policies.

See inside back cover or msp.org/pjm for submission instructions.

The subscription price for 2013 is US \$400/year for the electronic version, and \$485/year for print and electronic.

Subscriptions, requests for back issues and changes of subscribers address should be sent to Pacific Journal of Mathematics, P.O. Box 4163, Berkeley, CA 94704-0163, U.S.A. The Pacific Journal of Mathematics is indexed by Mathematical Reviews, Zentralblatt MATH, PASCAL CNRS Index, Referativnyi Zhurnal, Current Mathematical Publications and the Science Citation Index.

The Pacific Journal of Mathematics (ISSN 0030-8730) at the University of California, c/o Department of Mathematics, 798 Evans Hall \#3840, Berkeley, CA 94720-3840, is published twelve times a year. Periodical rate postage paid at Berkeley, CA 94704, and additional mailing offices. POSTMASTER: send address changes to Pacific Journal of Mathematics, P.O. Box 4163, Berkeley, CA 94704-0163.

PJM peer review and production are managed by EditFLOW ${ }^{\circledR}$ from Mathematical Sciences Publishers.

\section{PUBLISHED BY}

mathematical sciences publishers

nonprofit scientific publishing

http://msp.org/

(C) 2013 Mathematical Sciences Publishers 


\section{PACIFIC JOURNAL OF MATHEMATICS}

Volume $265 \quad$ No. $2 \quad$ October 2013

Singularity removability at branch points for Willmore surfaces

YANN BERNARD and TRISTAN RIVIÈRE

On Bach flat warped product Einstein manifolds

QIANG CHEN and CHENXU HE

On plane sextics with double singular points

Alex DeGTyareV

A computational approach to the Kostant-Sekiguchi correspondence

HEIKo DiETRICH and WILLEM A. DE GRAAF

Landau-Toeplitz theorems for slice regular functions over quaternions

GRAZIANO GENTILI and GiUlia SARFATTI

On surgery curves for genus-one slice knots

PATRICK M. GILMER and CHARLES LIVINGSTON

Characterizing abelian varieties by the reduction of the Mordell-Weil group

Chris Hall and Antonella Perucca

Variation of complex structures and the stability of Kähler-Ricci

Solitons

StUART J. HALl and ThOMAS MURPHY

On crossed homomorphisms of the volume preserving diffeomorphism groups

RYOJI KASAGAWA

Regularity at the boundary and tangential regularity of solutions of the Cauchy-Riemann system

TRAN VU KHANH and GIUSEPPE ZAMPIERI

On the Steinberg character of a semisimple $p$-adic group 